\title{
Impedance of a Cylindrical Dipole Having a Sinusoidal Current Distribution in a Homo- geneous Anisotropic Ionosphere
}

\author{
W. S. Ament, J. C. Katzin, M. Katzin, and B. Y.-C. Koo \\ Contribution from the Electromagnetic Research Corporation, College Park, Md.
}

(Received April 24, 1963; revised October 8, 1963)

\begin{abstract}
The input impedance of a cylindrical dipole in a homogeneous anisotropic ionosphere is determined for arbitrary values of the medium parameters and arbitrary orientation of the dipole with respect to the earth's magnetic field. A sinusoidal current distribution is assumed, as well as a low value of dipole excitation, so that the field equations may be assumed to be linear. The Green's function is expressed as a Fourier integral in spherical propagation-constant coordinates. In performing a residues evaluation of the radial integral in this coordinate system, it is found necessary to deform the contour differently in different angular regions. By an appropriate rotation of the coordinates, the boundaries of these angular regions are made to depend on a single angular variable. An expansion of the result in power series in the small parameter $\epsilon=$ (dipole radius/dipole half-length), in which only terms in $\log (1 / \epsilon)$ and $\epsilon^{0}$ are retained, leads to considerable simplification. It is found that the two dominant terms can be expressed as sine and cosine integrals, as in the free-space case, plus two finite single integrals which need numerical evaluation. The integrands of these latter integrals are expressed in terms of recursive routines.

The numerical integrations are in terms of the polar angle of the wave propagation vector with respect to the earth's magnetic field. Singularities of the integrand are encountered in the collisionless case when the applied frequency is below the plasma or gyrofrequencies. It is then necessary to carry out the numerical integration in the complex plane. Consequently the present numerical integration procedure is restricted to cases where this situation does not occur. Extensions of the treatment which remove these restrictions and methods of obtaining a more accurate current distribution are discussed briefly, the details being reserved for a later paper.

Numerical calculations of impedance for selected values of the parameters are exhibited in a set of curves.
\end{abstract}

\section{Introduction}

For several years, ionosphere probing by rocket-borne probes has used the technique of the antenna impedance probe [Jackson and Kane, 1959]. The impedance probe has the special merit that it is affected by the ionospheric parameters in the immediate vicinity of the proberoughly in the order of a wavelength or less of the probe excitation. Consequently, this type of probe gives local values of the parameters, rather than integrated values as in the Seddon [1953] type of experiment. It also can be operated at an excitation level low enough to avoid the creation of additional ionization in the medium by the rf field.

The proper interpretation of the impedance measurement of an antenna probe requires a knowledge of the input impedance as a function of the medium properties. The type of antenna used in rocket probing can be rather closely approximated as a cylindrical dipole. Fortunately one is interested in the impedance change produced by the ionized medium, so that deviations from the ideal cylindrical form can be expected to play a secondary role. For this reason, the subject of this paper is the calculation of the input impedance of a cylindrical dipole in a magneto-ionic medium.

Rocket experiments have shown that the antenna acquires a negative charge. This negative charge repels the electrons immediately around the antenna, with the result that the antenna environment is rendered inhomogeneous. This effect is a very important one from the standpoint of the application of the impedance probe technique to the ionosphere. If this type of

\footnotetext{
1 The research reported in this paper was supported by the National Aeronautics and Space Admfn 1stration under Contract NAS5-585.
} 
probe is to be useful, the nature of the inhomogeneity produced, and its effect on the antenna impedance must be capable of determination. The former of these two problems appears to be the more formidable one at present. In any event, a solution for the homogeneous case is a necessary first step, both to develop an insight to the nature and magnitude of the effects produced by various values of the medium parameters as well as to serve as a basis for a possible perturbation technique for the inhomogeneous distribution. The case of a homogeneous medium will be assumed in this paper.

The calculation of input impedance of a dipole is a straightforward problem when the distribution of current over the dipole is known. The current distribution is not arbitrary, however, since, in principle, it can be derived from Maxwell's equations and the known boundary conditions at the dipole surface. The key difficulty arises when one attempts to determine this current distribution from Maxwell's equations, since one is confronted with the problem of solving an integral equation [King, 1956]. Various iterative methods have been employed, but the accuracy of the result, as judged by comparison with experiment, is sensitive to the technique used.

In first approximation the current distribution along the dipole is sinusoidal. This approximation is quite good for very thin dipoles. The sinusoidal distribution may be considered to be the result of guided waves propagating in the medium along the outside of the conductor, at a velocity appropriate to the external medium, and perfectly reflected at the open ends, the interference between the two oppositely directed wave trains resulting in a standing wave with zero current at the outer ends.

Fortunately, as already mentioned, in the case of a dipole used as an impedance probe we are not interested in the exact calculation of the dipole impedance, but rather in the impedance change upon entry into the ionosphere. This change is thus a difference quantity, so that small deviations in the nature of end effects which are occasioned by the use of only an approximate current distribution can be expected to largely cancel out when the difference, or change, from the free-space value is formed. Furthermore, the results obtained on the basis of a sinusoidal current distribution may be made the basis for a variational procedure whereby the current distribution is optimized, so that the initial impedances values can be refined. In this paper we assume a sinusoidal current distribution, reserving the variational treatment for a subsequent communication.

A further assumption is made that the amplitude of the motion of the free electrons in the medium in response to the electric field of the dipole is so small that the refractive index is given by the standard Appleton-Hartree formula. This assumption makes the field equations linear, so that Fourier resolutions are admissible.

In the treatment given in this paper, the input impedance will be obtained by equating the complex power passing from the surface of the dipole into the medium to the complex power supplied to the dipole at its input terminals. In this formulation the Green's function is expressed as a Fourier integral in spherical propagation-constant coordinates. In performing a residues evaluation of the radial integral in this coordinate system, it is found necessary to deform the contour differently in different angular regions. By an appropriate rotation of the coordinates, the boundaries of these angular regions are made to depend on a single angular variable. An expansion of the result in power series in the small parameter $\epsilon=$ (dipole radius/ dipole half-length), in which only terms in $\log (1 / \epsilon)$ and $\epsilon^{0}$ are retained, leads to considerable simplification. It is found that the two dominant terms can be expressed as sine and cosine integrals, as in the free-space case, plus two finite single integrals which require numerical evaluation. The integrands of these latter integrals are expressed in terms of recursive routines.

The numerical integrations are in terms of the polar angle of the wave propagation vector with respect to the earth's magnetic field. Singular points of the integrand are encountered in the collisionless case when the applied frequency is below the plasma or gyrofrequencies. It is then necessary to carry out the numerical integration in the complex plane. Calculations given in this paper are restricted to cases where this situation does not occur. ${ }^{2}$ The closed-

\footnotetext{
2 This restriction will be removed in a subsequent paper.
} 
form terms, however, are valid in all parameter ranges.

Numerical calculations of impedance for selected values of the parameters are exhibited in a set of curves.

\section{Formulation of the Problem}

In view of the preceding discussion, we now undertake the calculation of the input impedance of a cylindrical dipole in an infinite homogeneous ionosphere having a constant superimposed magnetic field. This calculation is based on the following assumptions:

(a) The current distribution along the (center-fed) dipole is a sinusoidal function of distance along the dipole, being zero at the outer ends, and is uniform around the circumference of the dipole.

(b) In the calculation of the field at a typical point in space, the current can be considered as concentrated along the axis of the dipole.

(c) The ambient ionosphere is homogeneous, and its refractive indices are given by the Appleton-Hartree relations.

The dipole, of radius $r_{0}$ and length $2 l$, is considered to be fed at its center, with a current distribution as shown in figure 1.

We shall find it convenient to employ two coordinate systems, $\Sigma$ and $\Sigma^{\prime}$. Coordinate system $\Sigma$ has its $z$-axis along the earth's magnetic field, $H_{0}$. For $\Sigma^{\prime}$, the $z^{\prime}$-axis coincides with that of the dipole, and makes an angle $\theta \leq \pi / 2$ with $z$. The relative orientation of $\Sigma$ and $\Sigma^{\prime}$ is chosen so that the $z^{\prime}$-axis lies in the $y z$-plane. Thus the $x$-axis of $\Sigma$ and the $x^{\prime}$-axis of $\Sigma$ ' coincide. The orientations of the two sets of axes are shown in figure 2.

We shall assume a time dependence of $e^{i \omega t}$ for all field quantities and sources. Rationalized mks units will be used throughout.

The impedance will be determined by equating the complex power (i.e., volt-amperes) supplied to the antenna at its feed point to the complex power supplied by the dipole to the external medium, where the latter is obtained by integrating the normal component of the complex Poynting vector over the surface of the dipole. The result is

$$
Z=\frac{1}{\left|I^{2}\right|} \int_{s}\left(\mathbf{E} \times \mathbf{H}^{*}\right) \cdot d \mathbf{s} .
$$

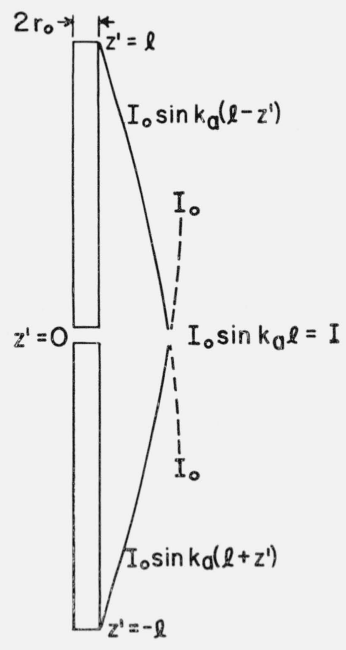

FIgURE 1. Illustrating dipole coordinates and current distribution.

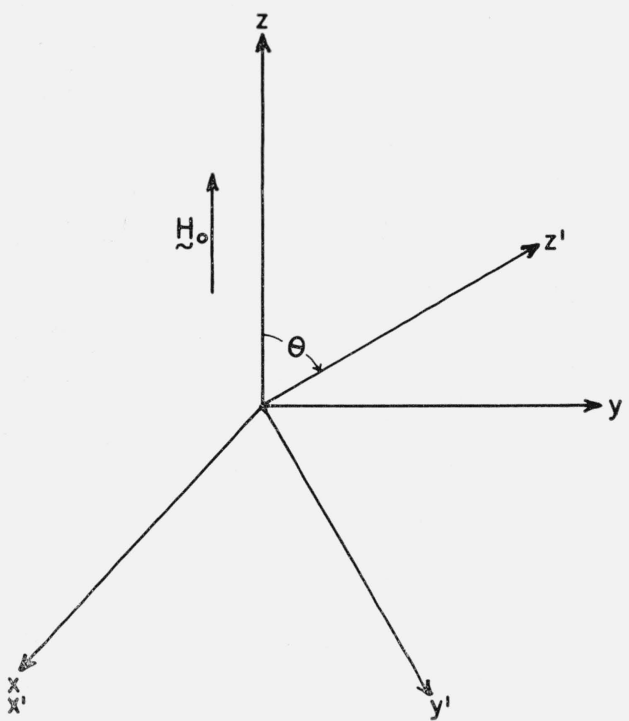

Figure 2. Coordinate systems. $x, y, z$ is a rectangular system oriented with the earth's magnetic field, while $x^{\prime}, y^{\prime}, z^{\prime}$ is oriented with the dipole axis along $z^{\prime}$. 
For the calculation of the integral in (1), we introduce cylindrical coordinates $\left(r^{\prime}, \varphi^{\prime}, z^{\prime}\right)$ in $\Sigma^{\prime}$, where $\varphi^{\prime}$ is measured from the $x^{\prime}$-axis. If we denote unit vectors by $\hat{e}$, then

$$
d \boldsymbol{s}=d A \hat{e}_{r^{\prime}} .
$$

The actual current flows on the surface $r^{\prime}=r_{0}$. However, for the calculation of the fields we make the usual assumption that the current is a line source on the axis of the cylinder, $r^{\prime}=0$, and directed along the axis. ${ }^{3} \quad$ Thus, if $j\left(x^{\prime}\right)$ represents this line current,

$$
H_{\mathrm{tang}}\left(r_{0}, \varphi^{\prime}, z^{\prime}\right)=\frac{1}{2 \pi r_{0}} \mathbf{j}\left(z^{\prime}\right) \times \hat{e}_{r^{\prime}}=\frac{1}{2 \pi r_{0}} j\left(z^{\prime}\right) \hat{e}_{\varphi^{\prime}} .
$$

On inserting (2) into the integrand of (1), we obtain

$$
Z=-\frac{1}{2 \pi\left|I^{2}\right|} \iint j\left(z^{\prime}\right)\left(E_{z} \cos \theta+E_{y} \sin \theta\right) d \varphi^{\prime} d z^{\prime} .
$$

The problem thus reduces to a determination of the electric field $E$. Once this has been found, the values of $E_{y}$ and $E_{z}$ can be inserted in (3), and this then evaluated to find the input impedance $Z$.

\section{Calculation of $\mathrm{E}$}

We now turn to the problem of finding the electric field $\mathbf{E}$. This obeys Maxwell's equations for the medium. In view of the free electrons and the superimposed static magnetic field of the earth, the medium is characterized by a dielectric tensor, so that a given component of field is due to current components in all three coordinate directions. On the assumption that the field equations are linear (implying sufficiently small vibrations of the free charges), Maxwell's equations guarantee that a solution must exist for $\mathbf{E}(\mathbf{x})$ of the form

$$
\mathbf{E}_{i}(\mathbf{x})=\int G_{i j}\left(\mathbf{x} \mid \mathbf{x}_{1}\right) J_{j}\left(\mathbf{x}_{1}\right) d^{3} x_{1}
$$

where $d^{3} x_{1}$ is a compact notation for $d x_{1 x} d x_{1 y} d x_{1 z}$, and the convention of summing over repeated indices is used.

In (4), $G_{i j}\left(\mathbf{x} \mid \mathbf{x}_{1}\right)$ is the $(i, j)$ component of the Green's tensor. Physically, it represents the electric field in the $i$ th direction at $\mathbf{x}$ due to the $j$ th component of the source current density $\mathbf{J}$ at $\mathbf{x}_{1}$. Thus $G_{i j}\left(\mathbf{x} \mid \mathbf{x}_{1}\right)$ propagates the effect of $\mathbf{J}$ at $\mathbf{x}_{1}$ to the point $\mathbf{x}$.

A derivation of the Green's function $G_{i j}\left(\mathbf{x x}_{1}\right)$ has been given by a number of authors (see, for example, Kogelnik [1960]). 'The result may be written in the form

From (4) and (5),

$$
G_{i j}\left(\mathbf{x} \mid \mathbf{x}_{1}\right)=-\frac{i \omega \mu_{0} k_{0}}{(2 \pi)^{3}} \int d^{3} q \frac{\Delta_{j i}(q)}{\Delta(q)} e^{-i \mathbf{q} \cdot \rho}
$$

$$
E_{i}(\mathbf{x})=-\frac{i \omega \mu_{0} k_{0}}{(2 \pi)^{3}} \int d^{3} x_{1} \int d^{3} q \frac{\Delta_{j i}(q)}{\Delta(q)} J_{j}\left(\mathbf{x}_{1}\right) e^{-i \mathbf{q} \cdot \rho}
$$

In (5) and (6), $\boldsymbol{\rho}$ is a numerical distance which is defined by

$k_{0}$ being the free-space wave number.

$$
\boldsymbol{\rho}=k_{0}\left(\mathbf{x}-\mathbf{x}_{1}\right)
$$

In $(5)$ and $(6), \Delta_{j i}$ is the $(j, i)$ th cofactor and $\Delta$ is the determinant of the following matrix:

$$
M(\mathbf{q})=\left(\begin{array}{lll}
q^{2}-q_{1}^{2}-\kappa_{11} & -q_{1} q_{2}-\kappa_{12} & -q_{1} q_{3}-\kappa_{13} \\
-q_{2} q_{1}-\kappa_{21} & q^{2}-q_{2}^{2}-\kappa_{22} & -q_{2} q_{3}-\kappa_{23} \\
-q_{3} q_{1}-\kappa_{31} & -q_{3} q_{2}-\kappa_{32} & q^{2}-q_{3}^{2}-\kappa_{33}
\end{array}\right)
$$

\footnotetext{
${ }^{3}$ It can be shown that this leads to errors of order no higher than terms neglected in the present treatment.
} 
in which

$$
q^{2}=q_{1}^{2}+q_{2}^{2}+q_{3}^{2}
$$

The $\kappa_{i j}$ are components of a tensor, which, in the coordinate system $\Sigma$, takes on the particularly simple form

where|

$$
\overline{\bar{\kappa}}=\left(\begin{array}{ccc}
\alpha_{1} & -i \alpha_{2} & 0 \\
i \alpha_{2} & \alpha_{1} & 0 \\
0 & 0 & \alpha_{3}
\end{array}\right)
$$

which are interrelated through

$$
\left.\begin{array}{l}
\alpha_{1}=1-\frac{x u}{u^{2}-y^{2}} \\
\alpha_{2}=\frac{x y}{u^{2}-y^{2}} \\
\alpha_{3}=1-\frac{x}{u}
\end{array}\right\}
$$

$x, y, z$ are the usual normalized (plasma frequency) ${ }^{2}$, gyrofrequency, and collision frequency, respectively:

and

$$
\left.\begin{array}{l}
x=\left(\omega_{N} / \omega\right)^{2} \\
y=\omega_{H} / \omega \\
z=\nu / \omega \\
u=1-i z
\end{array}\right\} .
$$

Then

$$
\Delta(\mathbf{q})=\operatorname{det}\{M(q)\}=-\alpha_{3}\left(q_{3}^{2}-\sigma_{1}^{2}\right)\left(q_{3}^{2}-\sigma_{2}^{2}\right)
$$

where $\sigma_{2}^{1}$ and $\sigma_{2}^{2}$ are the roots of the biquadratic

with

$$
\alpha_{3} \sigma^{4}-\left[2 \alpha_{1} \alpha_{3}-\left(\alpha_{1}+\alpha_{3}\right) p^{2}\right] \sigma^{2}+\left(p^{2}-\alpha_{3}\right)\left(\alpha_{1} p^{2}-\alpha_{1}^{2}+\alpha_{2}^{2}\right)=0
$$

These roots thus are given by

$$
p^{2}=q_{1}^{2}+q_{2}^{2}
$$

$$
\sigma_{1,2}^{2}=\frac{1}{2 \alpha_{3}}\left\{2 \alpha_{1} \alpha_{3}-\left(\alpha_{1}+\alpha_{3}\right) p^{2} \pm\left[\left(\alpha_{3}-\alpha_{1}\right)^{2} p^{4}-4 \alpha_{3} \alpha_{2}^{2} p^{2}+4 \alpha_{2}^{2} \alpha_{3}^{2}\right]^{1 / 2}\right\} .
$$

Since (3) does not involve $E_{x}$, so that $j_{x}$ is not required in (4), we need only four of the nine matrix elements of $G_{i j}\left(x \mid x_{1}\right)$ for the present problem. These are $G_{22}, G_{23}, G_{32}, G_{33}$. Then from (4) and $j_{z}=j_{z^{\prime}} \cos \theta, j_{y}=j_{z^{\prime}} \sin \theta$, (3) becomes

$$
\begin{aligned}
Z=-\frac{1}{2 \pi\left|I^{2}\right|} \int_{-l}^{l} d z^{\prime} \int_{0}^{2 \pi} d \varphi^{\prime} j^{*}\left(z^{\prime}\right) \int_{-l}^{l} d z_{1}^{\prime \prime} j\left(z_{1}^{\prime \prime}\right)\left\{\sin ^{2} \theta G_{22}\left(\mathbf{x}^{\prime} \mid \mathbf{x}_{1}^{\prime \prime}\right)\right. \\
\\
\left.\quad+\cos ^{2} \theta G_{33}\left(\mathbf{x}^{\prime} \mid \mathbf{x}_{1}^{\prime \prime}\right)+\sin \theta \cos \theta\left[G_{23}\left(\mathbf{x}^{\prime} \mid \mathbf{x}_{1}^{\prime \prime}\right)+G_{32}\left(\mathbf{x}^{\prime} \mid \mathbf{x}_{1}^{\prime \prime}\right)\right]\right\} .
\end{aligned}
$$

\section{Evaluation of $Z$}

To evaluate $Z$, we introduce dimensionless variables by putting

$$
\left.\begin{array}{l}
\Lambda=k_{\imath} l \\
\zeta=k_{a} z
\end{array}\right\}
$$


where $k_{a}$ is the propagation constant associated with the current distribution. For generality, we assume that the propagation constant $k_{a}$ in the current distribution may be complex, so that $\Lambda$ likewise may be complex. Then (17) becomes

$$
Z=-\frac{1}{2 \pi\left|k_{a}^{2}\right|\left|I^{2}\right|} \int_{-\Lambda}^{\Lambda} d \zeta^{\prime} \int_{0}^{2 \pi} d \varphi^{\prime} j^{*}\left(\zeta^{\prime}\right) \int_{-\Lambda}^{\Lambda} d \zeta_{1}^{\prime \prime} j\left(\zeta_{1}^{\prime \prime}\right)\left\{\sin ^{2} \theta G_{22}+\cos ^{2} \theta G_{33}+\sin \theta \cos \theta\left(G_{23}+G_{32}\right)\right\} .
$$

Formally, the final step is to substitute the required values of $G_{i j}\left(\mathbf{x}^{\prime} \mid \mathbf{x}^{\prime \prime}\right)$ from (5) into (19) and perform the integrations to obtain the value for $Z$. The quantity in braces in (19) involves the ratio $N(\mathbf{q}) / \Delta(\mathbf{q})$ where $\Delta(\mathbf{q})$ is given by (13), and $N(\mathbf{q})$ by

$$
N(\mathbf{q})=\sin ^{2} \theta\left[\left(q^{2}-\alpha_{1}\right)\left(q_{2}^{2}-\alpha_{3}\right)+q_{1}^{2}\left(\alpha_{3}-\alpha_{1}\right)\right]+\cos ^{2} \theta\left[\left(q^{2}-\alpha_{1}\right)\left(q_{3}^{2}-\alpha_{1}\right)-\alpha_{2}^{2}\right]+2 \sin \theta \cos \theta q_{2} q_{3}\left(q^{2}-\alpha_{1}\right) .
$$

Consequently (19) becomes

$$
Z=\frac{i \zeta_{0}}{(2 \pi)^{4}\left|I_{0}^{2}\right|}\left|\frac{k_{0}}{k_{a}}\right|^{2} \int d^{3} q \frac{N(\mathbf{q})}{\Delta(\mathbf{q})} \int_{-\Lambda}^{\Lambda} d \zeta^{\prime} \int_{-\Lambda}^{\Lambda} d \zeta_{1}^{\prime \prime} \int_{0}^{2 \pi} d \varphi^{\prime} j^{*}\left(\zeta^{\prime}\right) j\left(\zeta_{1}^{\prime \prime}\right) e^{-i \mathbf{q} \cdot \rho}
$$

in which $\zeta_{0}$ and $k_{0}$, respectively, are the free-space impedance and propagation constant.

We now are ready to introduce the assumption of a sinusoidal current distribution. We express this distribution, which is illustrated in figure 1 , in the form

$$
\left.\begin{array}{rlrl}
j(\zeta) & =I_{0} \sin (\Lambda-\zeta), & & 0 \leq \zeta \leq \Lambda, \\
& =I_{0} \sin (\Lambda+\zeta), & & 0 \geq \zeta \geq \Lambda
\end{array}\right\}
$$

and use this form for both $j^{*}\left(\zeta_{1}^{\prime}\right)$ and $j\left(\zeta_{2}^{\prime \prime}\right)$ in (17). As noted earlier, we assume that the propagation constant $k_{a}$ in the current distribution may be complex.

With the assumed form of current distribution (22), it is possible to carry out all the inner integrations in (21). The quantity $\rho$ in the exponent in (21) is the numerical distance between the current element on the axis at $\zeta_{1}^{\prime \prime}$ and the current element on the surface of the cylinder at $\zeta^{\prime}$. Hence

where

$$
\boldsymbol{q} \cdot \boldsymbol{\rho}=q_{z^{\prime}}\left(\zeta^{\prime}-\zeta_{1}^{\prime \prime}\right)+q_{y^{\prime}} R \sin \varphi^{\prime}+q_{x^{\prime}} R \cos \varphi^{\prime},
$$

$$
R=k_{0} r_{0}
$$

When the exponent in (21) becomes

By putting

$$
-i\left[q_{z^{\prime}}\left(\zeta^{\prime}-\zeta_{1}^{\prime \prime}\right)+R\left(q_{x^{\prime}} \cos \varphi^{\prime}+q_{y^{\prime}} \sin \varphi^{\prime}\right)\right] .
$$

$$
\begin{gathered}
q_{x^{\prime}} \cos \varphi^{\prime}+q_{y^{\prime}} \sin \varphi^{\prime}=\sqrt{q_{x^{\prime}}^{2}+q_{y^{\prime}}^{2}} \cos \left(\varphi^{\prime}+\gamma\right), \\
\gamma=\tan ^{-1}\left(q_{y^{\prime}} / q_{x^{\prime}}\right),
\end{gathered}
$$

the innermost integral in $(21)$ becomes, in view of the $2 \pi$-periodicity of the integrand

$$
\int_{0}^{2 \pi} e^{-i R\left(q_{x^{\prime}} \cos \varphi^{\prime}+q_{y^{\prime}} \sin \varphi^{\prime}\right)} d \varphi^{\prime}=2 \pi J_{0}\left(R \sqrt{q_{x^{\prime}}^{2}+q_{y^{\prime}}^{2}}\right) .
$$

In the $\Sigma$ system the Bessel function in (24) becomes

$$
J_{0}\left(R \sqrt{q_{1}^{2}+\left(q_{2} \cos \theta-q_{3} \sin \theta\right)^{2}} .\right.
$$

Although (24) has been evaluated in closed form, it will be necessary later on to revert to the integral form in carrying out a residues integration. 
The evaluation of the remaining integrals in (21) is straightforward, so that we obtain

in which

$$
Z=C \iint_{-\infty}^{\infty} \int_{\infty} \frac{N(\mathbf{q}) F(\mathbf{q})}{\Delta(\mathbf{q})} d q_{1} d q_{2} d q_{3}
$$

and

In $(27)$

in which

$$
C=\frac{i \zeta_{0}}{2 \pi^{3}\left|\sin ^{2} \Lambda\right|}
$$

$$
G=\frac{\Lambda}{\Lambda_{0}} \frac{\cos \Lambda_{0}\left(q_{3} \cos \theta+q_{2} \sin \theta\right)-\cos \Lambda}{\left(q_{3} \cos \theta+q_{2} \sin \theta\right)^{2}-\left(\Lambda / \Lambda_{0}\right)^{2}}
$$

$$
\Lambda_{0}=k_{0} l \text {. }
$$

Study of (26) shows that a transformation from rectangular $q$-coordinates to spherical coordinates is advantageous, since then the radicand in (18) does not pass through zero for real angles. The radial integration in this spherical coordinate system is then carried out by residues. One then gets analytically different results in different angular regions. In order to simplify the division of these regions, transformations (rotations) of the spherical coordinates are introduced in order that the regional boundaries depend on only one of the angular variables. The analytic forms of the integrand are then written out in full for each region, and further combinations of the component parts of the impedance expression can be made in order to simplify the subsequent manipulations.

The integrands are then studied as power series in the parameter $\epsilon \equiv$ (dipole radius/dipole half-length), which is a small number for practical dipoles. The dominant terms of these series are examined as functions of the angular variables. It is found that the two dominant terms can be expressed as sine and cosine integrals, as in the free-space case, plus two single integrals which need numerical evaluation. The integrands of these latter integrals can be evaluated recursively. It is believed that integrals similar to these are inescapable because of the form of the propagation constants in the magneto-ionic medium, which contain the angular variable under a radical.

In sections 6 and 7 , the consequences of assumptions (a) and (b) above are examined briefly. With respect to (a), it is pointed out that variational procedures can be formulated which lead to a determination of a more precise current distribution. With respect to (b), it is reported that this assumption causes no error in the dominant terms in $\epsilon$, retained in the present calculation.

Following the method of analysis outlined above, (25) is first transformed to spherical q-coordinates by introducing the following transformations:

$$
\left.\begin{array}{rl}
q_{1} & =q \sin \mu \cos \beta \\
q_{2} & =q \sin \mu \sin \beta \\
q_{3} & =q \cos \mu \\
q & =\left(q_{1}^{2}+q_{2}^{2}+q_{3}^{2}\right)^{1 / 2}
\end{array}\right\}
$$

It is evident from (3), (4), (11) and (12) that the integrand is an even function of $q$. Hence we can write

$$
\int_{0}^{\infty} \ldots q^{2} d q=\frac{1}{2} \int_{-\infty}^{\infty} \ldots q^{2} d q
$$


This will facilitate an evaluation of the $q$-integration by residues.

In the subsequent residues evaluation of the $q$-integration, it is necessary to expand the function $F(q)$ in $(25)$ into a form containing exponentials. The Bessel function in $F(q)$ can be written as (see $(24))$

$$
J_{0}(\quad)=\frac{1}{2 \pi} \int_{0}^{2 \pi} e^{-i R q \Phi} d \varphi
$$

where

$$
\Phi=\sin \mu \cos \beta \cos \varphi+(\sin \mu \sin \beta \cos \theta-\cos \mu \sin \theta) \sin \varphi .
$$

Using the transformations in (30), (28) becomes

where

$$
G=\Omega \frac{\cos \left(\Lambda_{0} \tau q\right)-\cos \left(\Lambda_{0} \Omega\right)}{\tau^{2} q^{2}-\Omega^{2}}
$$

$$
\begin{gathered}
\tau=\cos \mu \cos \theta+\sin \mu \sin \theta \sin \beta, \\
\Omega=\Lambda / \Lambda_{0} .
\end{gathered}
$$

If the above changes are introduced into (25), it becomes

$$
Z=\frac{C}{4 \pi} \int_{0}^{2 \pi} d \varphi \int_{0}^{2 \pi} d \beta \int_{0}^{\pi} \sin \mu d \mu \int_{-\infty}^{\infty} q^{2} \frac{N}{\Delta} G G^{*} e^{-i R \Phi q} d q
$$

The functions $\Phi$ and $G G^{*}$ control the convergence of the integrand at $|q| \rightarrow \infty$, and these depend on the angles $\varphi, \mu, \beta$ through (28B and (30). In order to simplify this angular dependence, we now make rotations of these coordinates in order that the boundaries of the regions of convergence depend on only one angular variable. This is accomplished with the transformations

$$
\left.\begin{array}{l}
\cos \mu \cos \theta+\sin \mu \sin \beta \sin \theta=\cos \sigma \\
\sin \mu \cos \beta=\sin \sigma \cos \psi \\
\sin \mu \sin \beta \cos \theta-\cos \mu \sin \theta=\sin \sigma \sin \psi
\end{array}\right\}
$$

followed by the transformations:

In this process

$$
\left.\begin{array}{l}
\cos \mu^{\prime}=\sin \sigma \sin \chi \\
\sin \mu^{\prime} \cos \beta^{\prime}=\sin \sigma \cos \chi \\
\sin \mu^{\prime} \sin \beta^{\prime}=\cos \sigma
\end{array}\right\} .
$$

$$
\int_{0}^{2 \pi} d \beta \int_{0}^{\pi} \sin \mu d \mu \text { changes to } \int_{0}^{2 \pi} d \beta^{\prime} \int_{0}^{\pi} \sin \mu^{\prime} d \mu^{\prime}
$$

Then (28) and (30) become, respectively,

$$
\begin{gathered}
\Phi=\sin \mu^{\prime} \cos \beta^{\prime} \\
\tau=\cos \sigma=\sin \mu^{\prime} \sin \beta^{\prime} .
\end{gathered}
$$

From (39) and (40), it is seen that $\Phi$ and $\tau$ have the same $\mu^{\prime}$-dependence, so that the convergence of the $q$-integration will depend only on the angle $\beta^{\prime}$.

The various coordinate transformations are depicted in figure 3.

Upon introducing (30) into (13), $\Delta$ may be written as

$$
\Delta=-\left[\alpha_{1}+\left(\alpha_{3}-\alpha_{1}\right) \cos ^{2} \mu\right]\left(q^{2}-r_{1}^{2}\right)\left(q^{2}-r_{2}^{2}\right),
$$




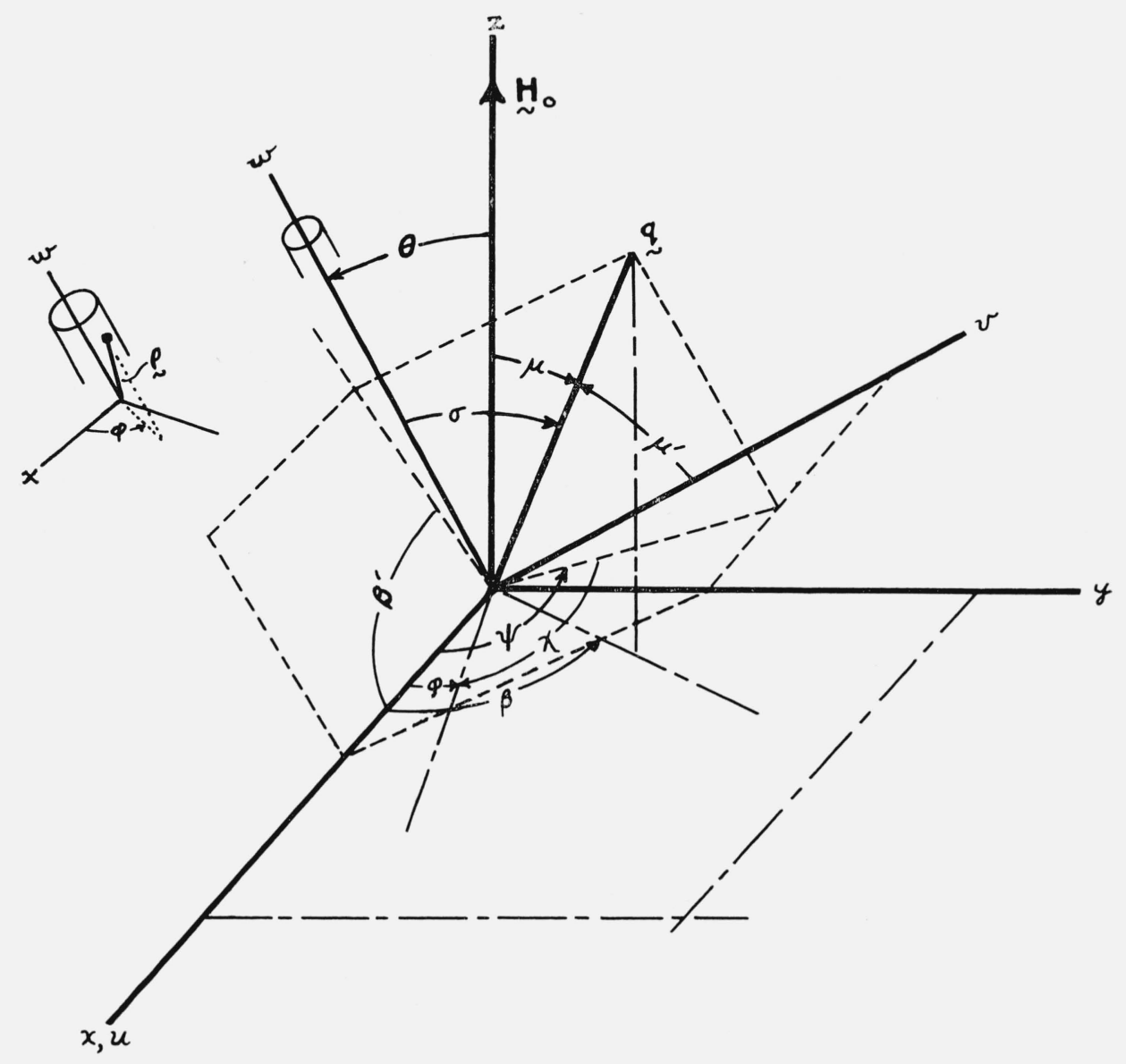

Figure 3. Coordinate system showing the various angles used in transforming variables.

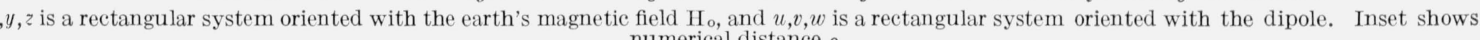
numerical distance $\rho$

where

$$
\begin{gathered}
r_{1,2}^{2}=\frac{2 \alpha_{1} \alpha_{3}+\left(\alpha_{1}-\alpha_{3}\right) \sin ^{2} \mu \pm \mathscr{R}}{2\left[\alpha_{1}+\left(\alpha_{3}-\alpha_{1}\right) \cos ^{2} \mu\right]} \\
\mathcal{R}=\left[\left(\alpha_{1}-\alpha_{3}\right)\left(\alpha_{1}-1\right)\left(4 \alpha_{3}^{2} \cos ^{2} \mu+\frac{\alpha_{1}-\alpha_{3}}{\alpha_{1}-1} \sin ^{4} \mu\right)\right]^{1 / 2} \\
=\frac{x y}{u^{2}-y^{2}}\left[4 \alpha_{3}^{2} \cos ^{2} \mu+\frac{y^{2}}{u^{2}} \sin ^{4} \mu\right]^{1 / 2} .
\end{gathered}
$$

In (43), the $x, y, u$ of (12) have been reintroduced for compression and in anticipation of numerical calculation. It should be noted that, for $u=1(z=0)$, the radicand is positive and has no zeros for real $\mu, 0<\mu<\pi / 2$. For future reference we write down

$$
\begin{aligned}
r_{1}^{2}+r_{2}^{2} & =\frac{2 \alpha_{1} \alpha_{3}+\left(\alpha_{1}-\alpha_{3}\right) \sin ^{2} \mu}{\alpha_{1}+\left(\alpha_{3}-\alpha_{1}\right) \cos ^{2} \mu} \\
r_{1}^{2} r_{2}^{2} & =\frac{\alpha_{3}\left(\alpha_{2} \alpha_{3}+\alpha_{1}-\alpha_{3}\right)}{\alpha_{1}+\left(\alpha_{3}-\alpha_{1}\right) \cos ^{2} \mu} .
\end{aligned}
$$

In a similar way, (20) for $N$ becomes

$$
N \equiv \mathcal{A} q^{4}-\mathscr{B} q^{2}+\mathcal{C},
$$

387 
where

$$
\begin{aligned}
\mathscr{A} & =\cos ^{2} \sigma=\tau^{2} \\
\mathscr{B} & =\alpha_{1} \cos ^{2} \sigma+\left(\alpha_{1}-\alpha_{3}\right) \sin ^{2} \theta \sin ^{2} \sigma \cos ^{2} \psi+\left(\alpha_{1} \cos ^{2} \theta+\alpha_{3} \sin ^{2} \theta\right) \\
& =\alpha_{1} \tau^{2}+\left(\alpha_{1}-\alpha_{3}\right) \sin ^{2} \theta \cos ^{2} \psi\left(1-\tau^{2}\right)+\left(\alpha_{1} \cos ^{2} \theta+\alpha_{3} \sin ^{2} \theta\right) \\
\mathcal{C} & =\alpha_{1} \alpha_{3}+\left(\alpha_{1}-\alpha_{3}\right) \cos ^{2} \theta
\end{aligned}
$$

with the above transformations, (36) takes the form

$$
Z=-\frac{C}{4 \pi} \int_{0}^{2 \pi} d \psi \int_{0}^{2 \pi} d \beta^{\prime} \int_{0}^{\pi} \frac{\sin \mu^{\prime} d \mu^{\prime}}{\alpha_{1}+\left(\alpha_{3}-\alpha_{1}\right) \cos ^{2} \mu} \int_{-\infty}^{\infty} \frac{q^{2} N G G^{*}}{\left(q^{2}-r_{1}^{2}\right)\left(q^{2}-r_{2}\right)^{2}} e^{-i R \Phi q} d q .
$$

In the $\mu^{\prime}, \beta^{\prime}$ variables,

$$
\begin{aligned}
\cos \mu & =\cos \sigma \cos \theta-\sin \sigma \sin \theta \sin \psi \\
& =\sin \mu^{\prime} \sin \beta^{\prime} \cos \theta-\sqrt{1-\sin ^{2} \mu^{\prime} \sin ^{2} \beta^{\prime}} \sin \theta \sin \psi,
\end{aligned}
$$

in which the radical is nonnegative, since $0 \leq \sigma \leq \pi$.

In order to perform a residues evaluation, it is necessary to split $G$ and $G^{*}$ into exponentials. For compactness, we write

Then

$$
\begin{aligned}
\xi & =R \sin \mu^{\prime} \cos \beta^{\prime}, \\
\eta & =\Lambda_{0} \sin \mu^{\prime} \sin \beta^{\prime}, \\
r_{3} & =\Lambda / \eta \\
r_{4} & =-\Lambda^{*} / \eta
\end{aligned}
$$

where

$$
G G^{*} e^{-i R \Phi q}=\frac{\left|\Omega^{2}\right|}{\tau^{4}} \frac{H(q)}{\left(q^{2}-r_{3}^{2}\right)\left(q^{2}-r_{4}^{2}\right)},
$$

$$
H(q)=\frac{1}{4}\left[e^{-i q(\xi+2 \eta)}+e^{-i q(\xi-2 \eta)}-\frac{1}{2}\left(\cos \Lambda+\cos \Lambda^{*}\right)\left[e^{-i q(\xi+\eta)}+e^{-i q(\xi-\eta)}\right]+\left(\frac{1}{2}+\cos \Lambda \cos \Lambda^{*}\right) e^{-i q \xi} .\right.
$$

Then (48) becomes

in which

$$
\mathrm{Z}=-\frac{C}{4 \pi} \int_{0}^{2 \pi} d \psi \int_{0}^{2 \pi} d \beta^{\prime} \int_{0}^{\pi} \frac{\sin \mu^{\prime} d \mu^{\prime}}{\alpha_{1}+\left(\alpha_{3}-\alpha_{1}\right) \cos ^{2} \mu} \cdot Q
$$

The factor

$$
Q=\frac{\left|\Omega^{2}\right|}{\tau^{4}} \int_{-\infty}^{\infty} \frac{q^{2} N(q) H(q) d q}{\left(q^{2}-r_{1}^{2}\right)\left(q^{2}-r_{2}^{2}\right)\left(q^{2}-r_{3}^{2}\right)\left(q^{2}-r_{4}^{2}\right)} \cdot
$$

$$
\alpha_{1}+\left(\alpha_{3}-\alpha_{1}\right) \cos ^{2} \mu
$$

in the denominator will be left in that form, since ultimately we shall return to the $\varphi, \mu, \beta$ coordinates in making use of this factor. To facilitate the evaluation of the $q$-integral of (54) by residues, we have written $H(q)$ in (52) in exponential form.

The poles $\pm r_{i}$ of (54) occur in pairs; the contour passes below $-r_{i}$ and above $+r_{i}$; this choice makes $e^{-i r_{i} D^{\prime}}$ an outgoing wave for distance $D^{\prime}>0$, since the $r_{i}$ have negative imaginary parts for $z>0$. Writing the integral so as to include all poles, the $q$-integration consequently yields

$$
Q=-2 \pi i \frac{\left|\Omega^{2}\right|}{\tau^{4}} \sum_{i=1}^{4} \frac{r_{i} N\left(r_{i}\right) H\left(r_{i}\right)}{2\left(r_{i}^{2}-r_{j}^{2}\right)\left(r_{i}^{2}-r_{k}^{2}\right)\left(r_{i}^{2}-r_{l}^{2}\right)}
$$

where $j, k, l$ with $j \neq k \neq l$, represent the three other subscripts possible after $i$ is chosen. $r_{i}$ is chosen as that root of $(42)$ which has negative imaginary part. $H\left(r_{i}\right)$, in view of the discussion 
above, is given by

$$
\begin{aligned}
H\left(r_{i}\right)=\frac{1}{4}\left[e^{-i r_{i}|\xi+2 \eta|}+e^{-i r_{i}|\xi-2 \eta|}\right]-\frac{1}{2}\left(\cos \Lambda+\cos \Lambda^{*}\right)\left[e^{-i r_{i}|\xi+\eta|}+e^{-i r_{i}|\xi-\eta|}\right] & \\
& +\left(\frac{1}{2}+\cos \Lambda \cos \Lambda^{*}\right) e^{-i r_{i}|\xi|} .
\end{aligned}
$$

The absolute value signs in the exponents require that $\mu^{\prime}$ and $\beta^{\prime}$ be real in the remaining in tegrations.

The relative values of $\xi$ and $\eta$ may be divided into three regions, in which the integration over $\beta^{\prime}$ must be treated differently. We define these by

$$
\begin{array}{ll}
\text { Region I: } & |\xi|>|2 \eta|, \\
\text { Region II: } & |2 \eta|>|\xi|>|\eta|, \\
\text { Region III: } & |\eta|>|\xi| .
\end{array}
$$

From (56), we may write after some rearrangement:

$$
\text { in region I, } \quad \begin{aligned}
H\left(r_{i}\right) & \equiv A\left(r_{i}\right)=e^{-i r_{i}|\xi|}\left(\cos \eta r_{i}-\cos \Lambda\right)\left(\cos \eta r_{i}-\cos \Lambda^{*}\right) \\
\text { in region II, } \quad H\left(r_{i}\right) & =A\left(r_{i}\right)+\frac{1}{2}\left[e^{-i r_{i}\left|\eta_{\eta}\right|} \cos \left(r_{i} \xi\right)-e^{-i r_{i}|\xi|} \cos \left(2 r_{i} \eta\right)\right] \\
& =A\left(r_{i}\right)-\frac{i}{2} \sin \left[r_{i}(|2 \eta|-|\xi|)\right] \\
& \equiv A\left(r_{i}\right)+B\left(r_{i}\right)
\end{aligned}
$$

in region III, $H\left(r_{i}\right)=A\left(r_{i}\right)+B\left(r_{i}\right)+i\left(\cos \Lambda+\cos \Lambda^{*}\right) \sin \left[r_{i}(|\eta|-|\xi|)\right]$

$$
\equiv A\left(r_{i}\right)+B\left(r_{i}\right)+C\left(r_{i}\right) .
$$

Symbolizing the angular integration over region I by $\int_{\mathrm{I}}$, etc., and the integrand by $S\left(r_{i}\right) H\left(r_{i}\right)$, we have

$$
\begin{aligned}
& Z=\int_{\mathrm{I}+\mathrm{II}+\mathrm{III}} A\left(r_{i}\right) S\left(r_{i}\right) d \beta^{\prime}+\int_{\mathrm{II}+\mathrm{III}} B\left(r_{i}\right) S\left(r_{i}\right) d \beta^{\prime}+\int_{\mathrm{III}} C\left(r_{i}\right) S\left(r_{i}\right) d \beta^{\prime} \\
& \equiv \quad Z_{1} \quad+\quad Z_{2} \quad+\quad Z_{3}
\end{aligned}
$$

The boundaries of the regions I, II, III, which depend on $\beta^{\prime}$, will be discussed in section 4.1.

\subsection{Evaluation of $Z_{1}$}

Since the regional sum $I+I I+I I I$ in $Z_{1}$ comprises the full range of angular variables of integration, we may conveniently rotate back to the original $\varphi, \mu, \beta$ variables in (36) to take advantage of the fact that the complication of the radical in (43) involves only $\mu$. Also we note here that $A\left(r_{i}\right) S\left(r_{i}\right)$ has no singularities for $i=3$, 4, so that $\sum_{i=1}^{4}$ in $(55)$ may be replaced by $\sum_{i=1}^{2}$ in evaluating $Z_{1}$. Hence we obtain

$$
\begin{aligned}
Z_{1}=C_{1} \int_{0}^{2 \pi} d \varphi \int_{0}^{2 \pi} d \beta \int_{0}^{\pi} \frac{\sin \mu d \mu}{\alpha_{1}+\left(\alpha_{3}-\alpha_{1}\right) \cos ^{2} \mu} & \\
& \frac{1}{r_{1}^{2}-r_{2}^{2}}\left\{r_{1} N\left(r_{1}\right)\left(\frac{\cos \eta r_{1}-\cos \Lambda}{\eta^{2}-\Lambda^{2}}\right)\left(\frac{\cos \eta r_{1}-\cos \Lambda^{*}}{\eta^{2}-\Lambda^{* 2}}\right) e^{-i r_{1}|\xi|}\right. \\
& \left.\quad-r_{2} N\left(r_{2}\right)\left(\frac{\cos \eta r_{2}-\cos \Lambda}{\eta^{2}-\Lambda^{2}}\right)\left(\frac{\cos \eta r_{2}-\cos \Lambda^{*}}{\eta^{2}-\Lambda^{* 2}}\right) e^{-i r_{2}|\xi|}\right\}
\end{aligned}
$$


in which

$$
\begin{aligned}
C_{1} & =\frac{\pi i \Lambda_{0}^{2}\left|\Lambda^{2}\right| C}{4 \pi} \\
& =-60\left(l / \lambda_{0}\right)^{2}\left|\frac{\Lambda}{\sin \Lambda}\right|^{2}
\end{aligned}
$$

Naturally, this form for $Z_{1}$ is obtainable from (36) by treating the two factors $G, G^{*}$ as entire functions whose growth far from the origin and from the poles is dominated by $e^{-i q \xi}$ for $|\xi|>0$.

Now the roots $r_{1}, r_{2}$ of $\Delta$ are numbers of order unity, and are exactly unity in the freespace case. The maximum value of $|\xi|$ is $R$, the dipole radius measured in free-space wavelengths, which is a small number for thin dipoles. In that case, then, we can expand in powers of $R$ and expect rapid convergence. The leading term in this expansion is obtained by setting $e^{-i r_{1,2}|\xi|}=1$. For this leading term, then, $\varphi$ vanishes from the integrand, so that the $\varphi$-integration yields a factor $2 \pi$.

Since there are functions of $\mu$ buried in the forms for $r_{1}, r_{2}$, no analytic $\mu$-integration is in sight. Hence we resort to numerical integration for this, restricting $\mu$ to real values. Since the $r_{i}$ do not involve $\beta$, only the $\mu$-integration needs to be done numerically.

In order to prevent the ( ) factors in (60) from assuming the form $\frac{0}{0}$ at some point in the $\beta, \mu$-range, we expand these factors in series. A typical factor may be written as

$$
\frac{\cos a-\cos \Lambda}{a^{2}-\Lambda^{2}}=\sum_{n=1}^{\infty} \frac{(-1)^{n}}{(2 n) !} \frac{a^{2 n}-\Lambda^{2 n}}{a^{2}-\Lambda^{2}}
$$

Then, after some manipulation, we obtain

$$
\left(\frac{\cos a-\cos \Lambda}{a^{2}-\Lambda^{2}}\right)\left(\frac{\cos a-\cos \Lambda^{*}}{a^{2}-\Lambda^{* 2}}\right)=\sum_{n=0}^{\infty} a^{2 n} D_{n}
$$

where

$$
D_{n}=(-1)^{n} \sum_{m=0}^{n} \sum_{k=0}^{\infty} \sum_{j=0}^{\infty} \frac{(-1)^{j+k} \Lambda^{2 j} \Lambda^{* 2 k}}{[2(m+j+1)] ![2(n-m+k+1)] !}
$$

$D_{n}$, which involves only the constants $\Lambda, \Lambda^{*}$, can be calculated and tabulated against $n$ in advance of the main calculation. Since

$$
a=r_{i} \eta=\Lambda_{0} r_{i} \cos \sigma=\Lambda_{0} r_{i}(\cos \mu \cos \theta+\sin \mu \sin \beta \sin \theta),
$$

the $a^{2 n}$ lead to terms containing integral powers of $\cos ^{2} \sigma$. Furthermore, as can be seen from (40) and (46), $N\left(r_{i}\right)$ involves $\sigma$ and $\beta$ in the forms $\cos ^{2} \sigma$ and $\cos ^{2} \beta$. Hence in the $\beta$-integration we need to evaluate

and

$$
P_{n}=\frac{1}{2 \pi} \int_{0}^{2 \pi} \cos ^{2 n} \sigma d \beta,
$$

$$
Q_{n}=\frac{1}{2 \pi} \int_{0}^{2 \pi} \cos ^{2} \beta \cos ^{2 n} \sigma d \beta .
$$

These may be evaluated to obtain

$$
\begin{aligned}
& P_{n}=\sum_{m=0}^{n} \frac{(2 n) !}{(2 m) ![2(n-m)] !}(\cos \mu \cos \theta)^{2(n-m)}(\sin \mu \sin \theta)^{2 m} x_{m} \\
& Q_{n}=\sum_{m=0}^{n} \frac{(2 n) !}{(2 m) ![2(n-m)] !}(\cos \mu \cos \theta)^{2(n-m)}(\sin \mu \sin \theta)^{2 m} y_{m}
\end{aligned}
$$


where

$$
x_{m}=\frac{(2 m) !}{2^{2 m} m ! m !}, \quad y_{m}=\frac{x_{m}}{2(m+1)} .
$$

$\left(x_{n}\right.$ may be calculated recursively from $z_{0}=1, x_{n}=\frac{2 n-1}{2 n} x_{n-1}$.)

With these results, we get from (60) on putting

and using (46) for $N\left(r_{i}\right)$,

$$
e^{-i r_{i}|\xi|}=1+0(R)
$$

$$
\begin{gathered}
Z_{1}=2 \pi C_{1} \int_{0}^{2 \pi} d \beta \int_{0}^{\pi} \frac{\sin \mu d \mu}{\alpha_{1}+\left(\alpha_{3}-\alpha_{1}\right) \cos ^{2} \mu} \cdot \sum_{i, j=1}^{2} \frac{\mathcal{A} r_{i}^{5}-\mathscr{B} r_{i}^{3}+\mathcal{C}\left(r_{i}\right.}{r_{i}^{2}-r_{j}^{2}} \cdot \sum_{n=0}^{i \infty}\left(r_{i} \eta\right)^{2 n} D_{n} \\
=2 \pi C_{1} \int_{0}^{2 \pi} d \beta \int_{0}^{\pi} \frac{\sin \mu d \mu}{\alpha_{1}+\left(\alpha_{3}-\alpha_{1}\right) \cos ^{2} \mu} \\
\cdot \sum_{n=0}^{\infty} \frac{\mathcal{A}\left(r_{1}^{2 n+5}-r_{2}^{2 n+5}\right)-\mathscr{B}\left(r_{1}^{2 n+3}-r_{2}^{2 n+3}\right)+\mathcal{C ( r _ { 2 } ^ { 2 n + 1 } - r _ { 2 } ^ { 2 n + 1 } )} \eta^{2 n} D_{n} .}{r_{1}^{2}-r_{2}^{2}}
\end{gathered}
$$

The summation contains terms of the form

where

$$
\frac{r_{1}^{2 n+1}-r_{2}^{2 n+1}}{r_{1}^{2}-r_{2}^{2}} \equiv \frac{c_{n}}{r_{1}+r_{2}}
$$

$$
\begin{aligned}
& c_{0}=1, c_{1}=r_{1}^{2}+r_{2}^{2} r_{2}+r_{2}^{2} \\
& c_{n}=\left(r_{1}^{2}+r_{2}^{2}\right) c_{n-1}-r_{1}^{2} r_{2}^{2} c_{n-2} .
\end{aligned}
$$

Hence, putting $\cos \mu=t$, we obtain

$$
\begin{aligned}
& Z_{1}=8 \pi^{2} C_{1} \int_{0}^{1} \frac{d t}{\alpha_{1}+\left(\alpha_{3}-\alpha_{1}\right) t^{2}} \frac{1}{r_{1}+r_{2}} \\
& \cdot \sum_{n=0}^{\infty} D_{n} \Lambda_{0}^{2 n}\left\{\left(c_{n+2}-\alpha_{1} c_{n+1}\right) P_{n+1}+\left[\left(\alpha_{3}-\alpha_{1}\right) \cos ^{2} \theta-\alpha_{3}\right] c_{n \times 1} P_{n}\right. \\
& \left.+\left[\alpha_{1} \alpha_{3}-\left(\alpha_{3}-\alpha_{1}\right) \cos ^{2} \theta\right] c_{n} P_{n}+\left(\alpha_{3}-\alpha_{1}\right) c_{n+1} \sin ^{2} \theta\left(1-t^{2}\right) Q_{n}\right\} .
\end{aligned}
$$

It should be noted that the appearance of $\theta$, both explicitly and implicitly in $P_{n}$, $Q_{n}$, is always as an even power of $\cos \theta$ or $\sin \theta$, so that

$$
Z_{1}(\theta)=Z_{1}(\pi-\theta),
$$

as is required physically. Equations (42), (63), (66), (67) and (69) permit the recursive evaluation of the integrand of (70) for each $\mu$. Thus what remains is a numerical integration over $\mu$.

\subsection{Evaluation of $Z_{2}$}

In $Z_{2}$ (and later in $Z_{3}$ ), we leave $\psi, \mu^{\prime}, \beta^{\prime}$ as the angular integration variables in order to have the boundaries of regions II and III determined by the single azimuthal variable $\beta^{\prime}$. Later, after introduction of suitable approximations, it will be possible to return to the original variables $\varphi, \mu, \beta$.

The integral $Z_{2}$ is taken over the region II $+\mathrm{III}$, which, from $(57 \mathrm{~b})$, is given by

By defining

$$
|\xi| \leq|2 \eta|
$$

$$
\epsilon_{1}=\frac{R}{2 \Lambda_{0}}=\frac{r_{0}}{2 l}=\frac{1}{2} \epsilon
$$


this can be written as

$$
\left|\tan \beta^{\prime}\right| \geq \epsilon .
$$

The result of the $q$-integration in (54) is given by (55). For $Z_{3}$, we have to insert for $H\left(r_{i}\right)$, in virtue of $(58 \mathrm{~b}, \mathrm{c})$.

$$
H\left(r_{i}\right)=B\left(r_{i}\right)=-\frac{i}{2} \sin \left[r_{i}(|2 \eta|-|\xi|)\right]=-\frac{i}{2} \sin \left[2 r_{i}|\eta|\left(1-\epsilon_{1}\left|\cot \beta^{\prime}\right|\right)\right] .
$$

It contributes to simplicity to write $Z_{2}$ in terms of the quantities

$$
S_{i}=r_{i}|\cos \sigma|=r_{i}\left|\sin \mu^{\prime} \sin \beta^{\prime}\right| .
$$

Then we have from $(50)$

$$
\left.\begin{array}{l}
S_{3}=\Lambda / \Lambda_{0}=\Omega \\
S_{4}=-\Lambda^{*} / \Lambda_{0}=-\Omega^{*}
\end{array}\right\}
$$

$S_{1}$ and $S_{2}$, in view of the dependence of $r_{1}$ and $r_{2}$ on $\mu$ (see (42)), and the explicit factor $\left|\sin \beta^{\prime}\right|$ in (74), are functions of $\beta^{\prime}$, while $S_{3}$ and $S_{4}$ are not. Then from (53), (55), and (58b, c), we have

where

$$
Z_{2}=\frac{1}{8} C\left|\Omega^{2}\right| \int_{0}^{2 \pi} d \psi \int_{0}^{\pi} d \mu^{\prime} \int_{\mathrm{II}+\mathrm{III}} \frac{G_{1} d \beta^{\prime}}{\left|\sin \beta^{\prime}\right|}
$$

$$
G_{1}=\frac{1}{\alpha_{1}+\left(\alpha_{3}-\alpha_{1}\right) \cos ^{2} \mu} \cdot \sum_{i=1}^{4} \frac{\overline{\mathscr{A}} S_{i}^{4}-\overline{\mathscr{B}} S_{i}^{2}-\overline{\mathcal{C}}}{\left(S_{i}^{2}-S_{j}^{2}\right)\left(S_{i}^{2}-S_{k}^{2}\right)\left(S_{i}^{2}-S_{l}^{2}\right)} S_{i} \sin \left[2 \Lambda_{0} S_{i}\left(1-\epsilon_{1} \cot \beta^{\prime}\right)\right],
$$

in which, with $\tau=\cos \sigma$ again,

$$
\left.\begin{array}{l}
\overline{\mathcal{A}}=\tau^{-2} \mathscr{A}=1 \\
\overline{\mathscr{B}}=\mathscr{B}=\alpha_{1} \tau^{2}+\left(\alpha_{1}-\alpha_{3}\right) \sin ^{2} \theta \cos ^{2} \psi\left(1-\tau^{2}\right)+\left(\alpha_{1} \cos ^{2} \theta+\alpha_{3} \sin ^{2} \theta\right) \\
\overline{\mathcal{C}}=\tau^{2} \mathcal{C}=\tau^{2}\left[\alpha_{1} \alpha_{3}+\left(\alpha_{1}-\alpha_{3}\right) \cos ^{2} \theta\right]
\end{array}\right\}
$$

The term $\left|\cot \beta^{\prime}\right|$ in the argument of $\sin [\quad]$ in (77) and the $\left|\sin \beta^{\prime}\right|$ in the denominator of (76) are both symmetric about each of the principal axes in the $\beta^{\prime}$-plane. As function of $S_{i}$, the summand in (77) expands in even powers of $S_{i}$, since $S_{i}$ sin $\left[2 \Lambda_{0} S_{i}\left(1-\epsilon_{1}\left|\cot \beta^{\prime}\right|\right)\right]$ is an even function of $S_{i}$ and the remaining terms are explicit in $S_{i}^{2}$. Thus, in the summand, the $\cos \sigma$ of (74) appears squared, and for this reason is symmetric about the principal axes in the $\beta$-plane. The same $\cos ^{2} \sigma$ symmetry appears in the factors $\overline{\mathcal{A}}, \overline{\mathcal{B}}, \overline{\mathcal{C}}$ as given in (78). There remains the dependence of $\cos \mu$ on $\beta^{\prime}$; this appears both in the explicit factor $1 /\left[\alpha_{1}+\left(\alpha_{3}-\alpha_{1}\right)\right.$ $\cos ^{2} \mu$ ] and implicitly in $S_{1}, S_{2}$ through their dependence on $r_{1}, r_{2}$, which in turn depend on $\mu$ through $\cos ^{2} \mu$, as is seen in (42). Now we may regard the integrand of (76) as expanded in powers of $\cos \mu$ or $\cos ^{2} \mu$; one sees from (49) that such an expansion will involve odd powers of $\cos \sigma=\sin \mu^{\prime} \sin \beta^{\prime}$, but that such odd powers of $\cos \sigma$ will be multiplied in turn by factors odd in $\sin \psi$. Now $\psi$ appears otherwise only in the $\cos ^{2} \psi$ term in $\bar{B}$; thus we may visualize doing the $\psi$-integral first, and we see that all terms in the expansion of the integrand having odd powers of $\sin \psi$ give zero contribution to $Z_{2}$. But these terms are exactly those which are odd in $\cos \sigma=\sin \mu^{\prime} \sin \beta^{\prime}$. 'Thus, in view of the $\psi$-integration, the terms in sin $\beta^{\prime}$ due to implicit and explicit dependence on $\cos \sigma$ are even, and therefore symmetric about each principal axis in the $\beta^{\prime}$-plane. Thus the entire integrand has this symmetry and we may henceforth confine $\beta^{\prime}$ to the first quadrant and drop the absolute value signs. Then in (76),

$$
\int_{\mathrm{II}+\mathrm{III}} \ldots d \beta^{\prime}=4 \int_{\tan -1 \epsilon_{1}}^{\pi / 2} \ldots d \beta^{\prime}
$$


The foregoing argument has also shown that part of the integrand of (76) which depends on the $S_{i}$ and on $\cos \mu$, either explicitly or via $r_{1}, r_{2}$, can be expanded in power series in $\cos \sigma=$ $\sin \mu^{\prime} \sin \beta^{\prime}$ and that this is a series in even powers of $\cos \sigma$, in view of the $\psi$-integration. Let us now regard this expansion as having been made. The $\beta^{\prime}$-dependence is then expressed in a series giving the $\beta^{\prime}$-integrals in the typical form

$$
\int_{\tan ^{-1} \epsilon_{1}}^{\pi / 2} \cos ^{2 n} \sigma\left(1-\epsilon_{1} \cot \beta^{\prime}\right)^{m} \frac{d \beta^{\prime}}{\sin \beta^{\prime}} .
$$

Expanding $\left(1-\epsilon_{1} \cot \beta^{\prime}\right)^{m}$ to get a double series, we have the typical integral

$$
I_{n, r}=\int_{\tan -1 \epsilon_{1}}^{\pi / 2}\left(\sin \beta^{\prime}\right)^{2 n}\left(\epsilon_{1} \cot \beta^{\prime}\right)^{r} d \beta^{\prime} / \sin \beta^{\prime} .
$$

Now we take advantage of the fact that we are dealing with a "thin" dipole, one in which $\epsilon=R / \Lambda_{0}$ is a small quantity. We wish to calculate terms of order $\log \left(1 / \epsilon_{1}\right)$ and $\epsilon_{1}^{0}$, and to neglect terms of order $\epsilon_{1}^{n}$ and $\epsilon_{1}^{n} \log \left(1 / \epsilon_{1}\right)$ for $n \geq 1$. By evaluating integrals of the type (81) with these considerations in mind, it may be shown that terms of the retained order occur only when $n=0$, or $r=0$, or both, the last alternative giving rise to a $\log \left(1 / \epsilon_{1}\right)$ term. We therefore write

$$
Z_{2}=Z_{2}^{\prime}+Z_{2}^{\prime \prime}+0\left[\epsilon_{1} \log \left(1 / \epsilon_{1}\right)\right]
$$

where $Z_{2}^{\prime}$ corresponds to $n=0$, and $Z_{2}^{\prime \prime}$ to $r=0, n \neq 0$. Thus $Z_{2}^{\prime}$ has the integrand obtained from (76) by putting $\cos \sigma=0$ in $S_{i}$, in $\cos \mu$, and in $\overline{\mathcal{A}}, \overline{\mathcal{B}}, \overline{\mathcal{C}}$, but retaining $\epsilon_{1} \cot \beta^{\prime}$ finite.

From (78), we see that $\bar{C}$ is of order $\cos ^{2} \sigma$, and from (74) that $S_{1}^{2}$ and $S_{2}^{2}$ are of this order. From (76), however, $S_{3}$ and $S_{4}$ are independent of $\cos \sigma$. Thus in the $\Sigma$ of $(77)$, for $i=1,2$ the numerator is of fourth order in $\cos \sigma$ and the denominator is of second order, so that the summands vanish for $i=1,2$ on setting $\cos \sigma=0$. Thus we obtain

$$
\begin{aligned}
Z_{2}^{\prime}=\frac{1}{2} C\left|\Omega^{2}\right| \int_{0}^{2 \pi} d \psi \int_{0}^{\pi} \frac{d \mu^{\prime}}{\alpha_{1}+\left(\alpha_{3}-\alpha_{1}\right) \cos ^{2} \mu_{0}} & \int_{\tan -1_{\epsilon_{1}}}^{\pi / 2} \frac{d \beta^{\prime}}{\sin \beta^{\prime}} \\
& \cdot \sum_{i=3}^{4} \frac{\left[\left(\overline{\mathscr{A}}_{0} S_{i}^{4}-\overline{\mathcal{B}}_{0} S_{i}^{2}\right)\right] \sin \left[2 \Lambda_{0} S_{i}\left(1-\epsilon_{1} \cot \beta^{\prime}\right)\right]}{S_{i}^{3}\left(S_{i}^{2}-S_{j}^{2}\right)},
\end{aligned}
$$

where, from $(50)$,

$$
\cos ^{2} \mu_{0}=\sin ^{2} \theta \sin ^{2} \psi
$$

and, from (78),

$$
\left.\begin{array}{l}
\overline{\mathscr{A}}_{0}=1 \\
\overline{\mathscr{B}}_{0}=\left(\alpha_{3}-\alpha_{1}\right) \sin ^{2} \theta \sin ^{2} \psi+\alpha_{1}=\alpha_{1}+\left(\alpha_{3}-\alpha_{1}\right) \cos ^{2} \mu_{0}
\end{array}\right\}
$$

We note that the integrand in (82) is free of $\mu^{\prime}$, and that the $\psi$-dependency, in virtue of (75), (83), and (84), is of the form

$$
\begin{aligned}
\int_{0}^{2 \pi} \frac{d \psi}{\alpha_{1}+\left(\alpha_{3}-\alpha_{1}\right) \sin ^{2} \theta \sin ^{2} \psi} & =\int_{0}^{2 \pi} \frac{d \psi}{\alpha_{1} \cos ^{2} \psi+\left(\alpha_{1} \cos ^{2} \theta+\alpha_{3} \sin ^{2} \theta\right) \sin ^{2} \psi} \\
& =2 \pi\left[\alpha_{1}\left(\alpha_{1} \cos ^{2} \theta+\alpha_{3} \sin ^{2} \theta\right)\right]^{-1 / 2},
\end{aligned}
$$

from Bierens de Haan [1957] table 47, No. 10. After integration over $\psi$ and $\mu^{\prime}$, (82) therefore becomes

$$
Z_{2}^{\prime}=\pi^{2} C\left|\Omega^{2}\right| \int_{\tan ^{-1} \epsilon_{1}}^{\pi / 2} \frac{d \beta^{\prime}}{\sin \beta^{\prime}} \cdot \sum_{i=3}^{4} \frac{\left[\alpha_{1}\left(\alpha_{1} \cos ^{2} \theta+\alpha_{3} \sin ^{2} \theta\right)\right]^{-1 / 2} S_{i}^{2}-1}{S_{i}\left(S_{i}^{2}-S_{j}^{2}\right)} \sin \left[2 \Lambda_{0} S_{2}\left(1-\epsilon_{1} \cot \beta^{\prime}\right)\right] .
$$

Writing

$$
g=\epsilon_{1} \cot \beta^{\prime},
$$


we now expand

$$
\sin \left[2 \Lambda_{0} S_{i}(1-g)\right]=\sin \left(2 \Lambda_{0} S_{i}\right) \cos \left(2 \Lambda_{0} S_{i} g\right)-\cos \left(2 \Lambda_{0} S_{i}\right) \sin \left(2 \Lambda_{0} S_{i} g\right)
$$

and further expand $\cos \left(2 \Lambda_{0} S_{i} g\right)$ and $\sin \left(2 \Lambda_{0} S_{i} g\right)$ in series. If we retain only terms of order $\log \left(1 / \epsilon_{1}\right)$ and $\epsilon_{1}^{0}$, as before, we may sum the resulting series to give the result

$$
\begin{aligned}
Z_{2}^{\prime}=\pi^{2} C\left|\Omega^{2}\right| \sum_{i=3}^{4} \frac{\left[\alpha_{1}\left(\alpha_{1} \cos ^{2} \theta+\alpha_{3} \sin ^{2} \theta\right)\right]^{-1 / 2} S_{i}^{2}-1}{S_{i}\left(S_{i}^{2}-S_{j}^{2}\right)} \\
\cdot\left\{\sin \left(2 \Lambda_{0} S_{i}\right)\left[C i\left(2 \Lambda_{0} S_{i}\right)+\log \frac{1}{\gamma \epsilon_{1} \Lambda_{0} S_{i}}\right]-\cos \left(2 \Lambda_{0} S_{i}\right) S i\left(2 \Lambda_{0} S_{i}\right)\right\},
\end{aligned}
$$

where $S i(x)$ and $C i(x)$ are the sine and cosine integrals [Jahnke and Emde, 1945], respectively, and in which use has been made of (71). This gives a closed form expression for $Z_{2}^{\prime}$.

On the other hand, $Z_{2}^{\prime \prime}$ has the integrand obtained from (76) by setting $\epsilon_{1} \cot \beta^{\prime}=0$ in the final $\sin [$ ] factor, so that

where

$$
Z_{2}^{\prime \prime}=\frac{1}{2} C\left|\Omega^{2}\right| \int_{0}^{2 \pi} d \psi \int_{0}^{\pi} d \mu^{\prime} \int_{\tan ^{-1} \epsilon_{1}}^{\pi / 2} \frac{G_{0}}{\alpha_{1}+\left(\alpha_{3}-\alpha_{1}\right) \cos ^{2} \mu} \frac{d \beta^{\prime}}{\sin \beta^{\prime}},
$$

$$
G_{0}=\sum_{i=1}^{4} \frac{\left(\overline{\mathscr{A}} S_{i}^{4}-\overline{\mathscr{B}} S_{i}^{2}+\overline{\mathcal{C}}\right) S_{i} \sin \left(2 \Lambda_{0} S_{i}\right)}{\left(S_{i}^{2}-S_{j}^{2}\right)\left(S_{i}^{2}-S_{k}^{2}\right)\left(S_{i}^{2}-S_{l}^{2}\right)}
$$

$G_{0}$ now is to be expanded in a series in powers of $\cos ^{2} \sigma$, starting with the first power (since the term in $\left(\cos ^{2} \sigma\right)^{0}$ already has been included in $\left.Z_{2}^{\prime}\right)$. Thus in $Z_{2}^{\prime \prime}$ the integrand has the leading term $\cos ^{2} \sigma=\sin ^{2} \mu^{\prime} \sin ^{2} \beta^{\prime}$. This and all higher terms vanish like $\sin ^{2} \beta^{\prime}$ or faster near $\beta^{\prime}=0$ so that, even with the $1 / \sin \beta^{\prime}$ factor, the $Z_{2}^{\prime \prime}$ integrand vanishes at $\beta^{\prime}=0$. This in turn permits the replacement of $\tan ^{-1} \epsilon_{1}$ by 0 as the lower limit of the $\beta^{\prime}$-integral, since this introduces a negligible error of order $\epsilon_{1}^{2}$. Hence (87) becomes

$$
Z_{2}^{\prime \prime}=\frac{1}{2} C\left|\Omega^{2}\right| \int_{0}^{2 \pi} d \psi \int_{0}^{\pi} d \mu^{\prime} \int_{0}^{\pi / 2} \frac{G_{0}}{\alpha_{1}+\left(\alpha_{3}-\alpha_{1}\right) \cos ^{2} \mu} \frac{d \beta^{\prime}}{\sin \beta^{\prime}}
$$

After expansion of $G_{0}$ in powers of $\cos ^{2} \sigma=\sin ^{2} \mu^{\prime} \sin ^{2} \beta^{\prime}$, we can make use of (38) and (37) to rotate back to the original coordinates $\varphi, \mu, \beta$. This is desirable because then $r_{1}, r_{2}$ depend only on $\mu$. The result is

$$
Z_{2}^{\prime \prime}=\frac{\pi}{4} C\left|\Omega^{2}\right| \int_{0}^{2 \pi} d \beta \int_{0}^{\pi} \frac{G_{0}}{|\cos \sigma|} \frac{\sin \mu d \mu}{\alpha_{1}+\left(\alpha_{3}-\alpha_{1}\right) \cos ^{2} \mu} .
$$

$G_{0}$ in (89) is a symmetric function of the four $S_{i}$. By expanding $\sin \left(2 \Lambda_{0} S_{i}\right)$ in a series, $G_{0}$ becomes

$G_{0}=\sum_{i=1}^{4} \frac{\left(\overline{\mathcal{A}} S_{i}^{4}-\overline{\mathscr{B}} S_{i}^{2}+\overline{\mathcal{C}}\right) S_{i} \sin \left(2 \Lambda_{0} S_{i}\right)}{\left(S_{i}^{2}-S_{j}^{2}\right)\left(S_{i}^{2}-S_{k}^{2}\right)\left(S_{i}^{2}-S_{l}^{2}\right)}=\sum_{i=1}^{4} \sum_{n=0}^{\infty} \frac{(-1)^{n}\left(2 \Lambda_{0}\right)^{2 n+1}}{(2 n+1) !} \frac{\overline{\mathcal{A}} S_{i}^{6}-\overline{\mathscr{B}} S_{i}^{4}+\overline{\mathcal{C}} S_{i}^{2}}{\left(S_{i}^{2}-S_{j}^{2}\right)\left(S_{i}^{2}-S_{k}^{2}\right)\left(S_{i}^{2}-S_{l}^{2}\right)} S_{i}^{2 n}$.

We can now make use of the results of symmetric function theory to obtain the desired expansion of $G_{0}$ in terms of $\cos ^{2} \sigma$. When this is done, the $\beta$-integration $\int_{0}^{2 \pi}|\cos \sigma|^{2 n+1} d \beta$ can be developed recursively, leaving only a $\mu$-integration to be performed numerically.

The expansion of a typical term of (91)

$$
\sum_{i=1}^{4} \frac{S_{i}^{2(n+N)}}{\left(S_{i}^{2}-S_{j}^{2}\right)\left(S_{i}^{2}-S_{k}^{2}\right)\left(S_{i}^{2}-S_{l}^{2}\right)}=A_{2 n+2 N}
$$


is expressed recursively in terms of the coefficients $A_{2 n}$ of appendix $\mathrm{A}$ as follows:

$$
\left.\begin{array}{c}
A_{0}=A_{2}=A_{4}=0, \quad A_{6}=1, \\
A_{2 n}=d_{1} A_{2 n-2}-d_{2} A_{2 n-4}+d_{3} A_{2 n-6}-d_{4} A_{2 n-8}
\end{array}\right\}
$$

where

$$
\begin{array}{ll}
\rho_{0}=\Omega^{2}+\Omega^{* 2}, & \rho_{2}=\Omega^{2} \Omega^{* 2}=\left|\Omega^{4}\right|, \\
\rho_{1}=r_{1}^{2}+r_{2}^{2}, & \rho_{3}=r_{1}^{2} r_{2}^{2} .
\end{array}
$$

It is evident that the $A_{2 n}$ will involve $\cos ^{2} \sigma$, in general. We therefore write

Hence we have from (92) and (93)

$$
A_{2 n}=\sum_{n=0}^{\infty} a_{m, n} \cos ^{2 m} \sigma .
$$

$$
\left.\begin{array}{rl}
a_{m, 0}= & a_{m, 1}=a_{m, 2}=0, \quad \text { for all } m \\
a_{0,3}=1, \quad a_{m, 3}=0 \quad \text { for } m \neq 0 \\
a_{m, n}= & \rho_{0} a_{m, n+1}-\rho_{2} a_{m, n-2}+\rho_{1} a_{m-1, n-1}-\rho_{0} \rho_{1} a_{m-1, n-2} \\
& +\rho_{1} \rho_{2} a_{m-1, n-3}-\rho_{3} a_{m-2, n-2}+\rho_{0} \rho_{3} a_{m-2, n-3}-\rho_{2} \rho_{3} a_{m-2, n-4}
\end{array}\right\}
$$

Then (91), together with (78), becomes

$$
\begin{aligned}
G_{0}=\sum_{m=1}^{\infty} \sum_{n=0}^{\infty} & (-1)^{n} \frac{\left(2 \Lambda_{0}\right)^{2 n+1}}{(2 n+1) !} \cos ^{2 m} \sigma \cdot\left\{a_{m, n+3}-a_{m, n+2}\left(\alpha_{1} \cos ^{2} \theta+\alpha_{3} \sin ^{2} \theta\right)\right. \\
& \left.-a_{m, n+2}\left(\alpha_{1}-\alpha_{3}\right) \sin ^{2} \theta \sin ^{2} \sigma \cos ^{2} \psi-a_{m-1, n+2} \alpha_{1}+a_{m-1, n+1}\left[\alpha_{1} \alpha_{3}+\left(\alpha_{1}-\alpha_{3}\right) \cos ^{2} \theta\right]\right\} .
\end{aligned}
$$

In $(96)$, the factor $\sin ^{2} \sigma \cos ^{2} \psi$ has been retained in order to facilitate the rotation back to the original $(\varphi, \mu, \beta)$ coordinates. By the second equation of (37), this factor then becomes $\sin ^{2} \mu \cos ^{2} \beta$. Hence, upon rotation of coordinates, (89) becomes, in virtue of $(90)$,

$$
\begin{aligned}
Z_{2}^{\prime \prime}=\frac{\pi C}{4}\left|\Omega^{2}\right| & \int_{0}^{2 \pi} d \beta \int_{0}^{\pi} \frac{\sin \mu d \mu}{\alpha_{1}+\left(\alpha_{3}-\alpha_{1}\right) \cos ^{2} \mu} \\
& \cdot \sum_{n=0}^{\infty} \sum_{m=1}^{\infty}(-1)^{n} \frac{\left(2 \Lambda_{0}\right)^{2 n+1}}{(2 n+1) !}|\cos \sigma|^{2 m-1} \cdot\left\{a_{m, n+3}-a_{m, n+2}\left(\alpha_{1} \cos ^{2} \theta+\alpha_{3} \sin ^{2} \theta\right)\right. \\
& \left.\quad-a_{m, n+2}\left(\alpha_{1}-\alpha_{3}\right) \sin ^{2} \theta \sin ^{2} \mu \cos ^{2} \beta-a_{m-1, n+2} \alpha_{1}+a_{m-1, n+1}\left[\alpha_{1} \alpha_{3}+\left(\alpha_{1}-\alpha_{3}\right) \cos ^{2} \theta\right]\right\} .
\end{aligned}
$$

Since from (34)

$$
\cos \sigma=\tau=\cos \mu \cos \theta+\sin \mu \sin \theta \sin \beta,
$$

the $\beta$-integration involves integrals of the form

$$
\begin{aligned}
& f_{n}=\frac{1}{2 \pi} \int_{0}^{2 \pi}|a+b \sin \beta|^{n} d \beta, \\
& g_{n}=\frac{1}{2 \pi} \int_{0}^{2 \pi} \cos ^{2} \beta|a+b \sin \beta|^{n} d \beta .
\end{aligned}
$$


These are evaluated in appendix B, again in terms of a recursive routine. Making use of the results obtained there, and making the substitution $\cos \mu=t,(97)$ becomes

$$
\begin{aligned}
Z_{2}^{\prime \prime}=\pi^{2} C\left|\Omega^{2}\right| \int_{0}^{1} & \frac{d t}{\alpha_{1}+\left(\alpha_{3}-\alpha_{1}\right) t^{2}} \cdot \sum_{n=0}^{\infty} \sum_{m=1}^{\infty}(-1)^{n} \frac{\left(2 \Lambda_{0}\right)^{2 n+1}}{(2 n+1) !} \\
& \cdot\left\{\left[a_{m, n+3}-a_{m, n+2}\left(\alpha_{1} \cos ^{2} \theta+\alpha_{3} \sin ^{2} \theta\right)-a_{m-1, n+2} \alpha_{1}+a_{m-1, n+1}\left(\alpha_{1} \alpha_{3}\right.\right.\right. \\
& \left.\left.\left.\quad+\left(\alpha_{1}-\alpha_{3}\right) \cos ^{2} \theta\right)\right] f_{2 m-1}+a_{m, n+2}\left(\alpha_{3}-\alpha_{1}\right) \sin ^{2} \theta\left(1-t^{2}\right) g_{2 m-1}\right\} .
\end{aligned}
$$

The final step in the evaluation of $Z_{2}^{\prime \prime}$ thus is a numerical integration over $t$.

\subsection{Evaluation of $Z_{3}$}

The evaluation of $Z_{3}$ follows a procedure entirely parallel to that for $Z_{2}$, it being only necessary to use

$$
\epsilon=R_{0} / \Lambda_{0}
$$

instead of $\epsilon_{1}$, and to use the appropriate value of $H\left(r_{i}\right)$ from (59). It is easily seen that the only changes required from the procedure of section 4.2 is to replace $2 \Lambda_{0}$ by $\Lambda_{0}$ everywhere, and to multiply the result by the coefficient

Hence, writing:

$$
-2\left(\cos \Lambda+\cos \Lambda^{*}\right) \text {. }
$$

$$
Z_{3}=Z_{3}^{\prime}+Z_{3}^{\prime \prime}
$$

we obtain from (86) and (98), respectively,

$$
\begin{gathered}
Z_{3}^{\prime}=-2 \pi^{2} C\left|\Omega^{2}\right|\left(\cos \Lambda+\cos \Lambda^{*}\right) \sum_{i=3}^{4} \frac{\left[\alpha_{1}\left(\alpha_{1} \cos ^{2} \theta+\alpha_{3} \sin ^{2} \theta\right)\right]^{-1 / 2} S_{i}^{2}-1}{S_{i}\left(S_{i}^{2}-S_{j}^{2}\right)} \\
\cdot\left\{\sin \left(\Lambda_{0} S_{i}\right)\left[C_{i}\left(\Lambda_{0} S_{i}\right)+\log \frac{1}{\gamma \in \Lambda_{0} S_{i}}\right]-\cos \left(\Lambda_{0} S_{i}\right) S_{i}\left(\Lambda_{0} S_{i}\right)\right\} . \\
\begin{aligned}
Z_{3}^{\prime \prime}=-2 \pi^{2} C\left|\Omega^{2}\right|\left(\cos \Lambda+\cos \Lambda^{*}\right) \int_{0}^{1} \frac{d t}{\alpha_{1}+\left(\alpha_{3}-\alpha_{1}\right) t^{2}} \cdot \sum_{n=0}^{\infty} \sum_{m=1}^{\infty}(-1)^{n} \frac{\Lambda_{0}^{2 n+1}}{(2 n+1) !} \\
\cdot\left\{\left[a_{m, n+3}-a_{m, n+2}\left(\alpha_{1} \cos ^{2} \theta+\alpha_{3} \sin ^{2} \theta\right)-a_{m-1, n+2} \alpha_{1}\right.\right. \\
\left.\left.+a_{m-1, n+1}\left(\alpha_{1} \alpha_{3}-\left(\alpha_{3}-\alpha_{1}\right) \cos ^{2} \theta\right)\right] f_{2 m-1}+a_{m, n+2}\left(\alpha_{3}-\alpha_{1}\right) \sin ^{2} \theta\left(1-t^{2}\right) g_{2 m-1}\right\} .
\end{aligned}
\end{gathered}
$$

\section{Limitations of the Numerical Integration}

The numerical integrations to be performed in (86), (98), (99), and (100) in the variable $t=\cos \mu$ are carried along the real $t$-axis over the range $0 \leq t \leq 1$. From figure 3 , it is seen that $\mu$ is the angle of propagation of the plane wave with respect to the earth's magnetic field. In the integrand, the term

$$
D \equiv \alpha_{1}+\left(\alpha_{3}-\alpha_{1}\right) t^{2}
$$

in the denominator vanishes, when $u=1$, at some value in the range $0 \leq t \leq 1$ when

This is equivalent to

$$
\alpha_{1} \alpha_{3} \leq 0
$$

$$
P \equiv(1-x)\left(1-y^{2}\right)\left(1-x-y^{2}\right) \leq 0 .
$$

When $D=0$, the present numerical procedure does not give meaningful results. This is due to the fact that when the quantity $\Delta$ of (41), which is the denominator of the Green's function in (36), vanishes, so that the Green's function becomes singular at some point of the integration path. This difficulty does not occur when the collision parameter $z$ is substantial, since then $D$ vanishes well off the real $t$-axis. For small $z$, or for $P$ positive and small, a finer integration grid will also give valid results. 
In order to a void the singularity and obtain a single numerical process valid in all parameter ranges, it is necessary to carry out the numerical integration essentially in the complex $t$-plane.* This will be postponed to a later treatment.

The relations for the impedance components developed in this report, namely (60), (86), (98), (99), and (100), have been programmed for the IBM 7090 computer. The program has been satisfactorily checked against the free-space impedance in the high-frequency limit. Numerical calculations have been made for certain parameter values. These will be discussed in section 8 .

\section{Propagation Constant $k_{a}$ and the Current Distribution}

As mentioned in section 4, the propagation constant of the assumed sinusoidal distribution of current along the dipole has been taken to be complex. This is reflected in the parameters

and

$$
\Lambda=k_{a} l
$$

$$
\Omega=\Lambda / \Lambda_{0},
$$

which, consequently, are complex, in general.

Our analysis tells us nothing about $k_{a}$, since we have assumed the form of the current distribution, which amounts to assuming that we know $k_{a}$. For trial numerical calculations, we have chosen values for $k_{a}$ near the average (over propagation direction) of the ordinary and extraordinary wave propagation constants. The value actually used was $k_{a}=\sqrt{1-x} k_{0}$.

In examining procedures for an optimum choice of $k_{a}$, we have been led to a variational formulation of the thin dipole impedance problem. This type of procedure, introduced by Storer [1950] in the free-space case, leads to a method for finding optimum values for the current distribution, which is then used to obtain an even more accurate impedance estimate. Furthermore, no major changes in the present analytical or numerical methods are required. These developments will be given in a later paper.

\section{Further Developments}

Our formulation is based on a Fourier integral representation of the anisotropic Green's function. Considerable effort has been expended, both by us and others, toward obtaining"a closed-form representation of this Green's function. We have been able to show, however, that such attempts are in vain, and that a representation, such as the Fourier integral used here, is a necessity.

Because we use a Fourier representation for the Green's function rather than a closed form as in the free-space impedance calculation, we have been able to evaluate precisely the error introduced by assumption (b) of section 4, namely, that the current can be considered as concentrated along the axis of the dipole in the calculation of the field at a typical point in space. It turns out that the corrections for this assumption occur only in the neglected terms of order $\epsilon$ or $\epsilon \log (1 / \epsilon)$ or higher, so that this assumption causes no error in the dominant terms retained in this paper.

Details of these developments will be given in a subsequent paper.

\section{Numerical Calculations}

In order to display the magnitude of the impedance as a function of the parameters, computations of $Z$ were made for several frequencies. These were calculated for frequencies of 10 , 5 , and $2 \mathrm{Mc} / \mathrm{s}$ and a set of ionospheric and antenna parameters supplied by Dr. R. G. Stone of the National Aeronautics and Space Administration. Table 1 lists the ionospheric parameters at the altitudes of interest. The corresponding values of $x, y, z$ are plotted in figures 4 and 5 for 10 and $5 \mathrm{Mc} / \mathrm{s}$, respectively. From these it is seen that in an altitude region around $300 \mathrm{~km}$, and of width increasing with decreasing frequency the parameter $x$ becomes comparable with

\footnotetext{
*Note added in proof:

Suitable patbs in the complex $t$-plane have been found for the numerical integrations $Z_{1}, Z_{2}^{\prime \prime}$, and $Z_{3}^{\prime \prime}$. With a 16-point Gaussian numerical integration procedure, calculations have been carried out at frequencies as low as about $2000 \mathrm{c} / \mathrm{s}$ without difficulty.
} 
TABLE 1

\begin{tabular}{|c|c|c|c|}
\hline Altitude & $\begin{array}{l}\text { Electron } \\
\text { density }\end{array}$ & Mag. field & $\begin{array}{l}\text { Collision } \\
\text { frequency }\end{array}$ \\
\hline $\begin{array}{l}k m \\
100 \\
150 \\
200 \\
250 \\
300\end{array}$ & $\begin{array}{c}\text { per } \mathrm{cm}^{3} \\
1.2 \times 10^{5} \\
1.7 \\
2.5 \\
3.5 \\
5.2\end{array}$ & $\begin{array}{c}\text { Gauss } \\
0.52 \\
.51 \\
.49 \\
.48 \\
.47\end{array}$ & $\begin{array}{r}s e c^{-1} \\
1000 \\
100 \\
500 \\
1000 \\
920\end{array}$ \\
\hline $\begin{array}{l}350 \\
400 \\
450 \\
500 \\
550\end{array}$ & $\begin{array}{c}4.3 \\
2.7 \\
1.8 \\
1.3 \\
8.3 \times 10^{4}\end{array}$ & $\begin{array}{l}.46 \\
.45 \\
.44 \\
.43 \\
.42\end{array}$ & $\begin{array}{l}840 \\
750 \\
670 \\
590 \\
510\end{array}$ \\
\hline $\begin{array}{l}600 \\
650 \\
700 \\
750 \\
800\end{array}$ & $\begin{array}{l}5.7 \\
4.0 \\
2.9 \\
2.2 \\
1.7\end{array}$ & $\begin{array}{l}.41 \\
.40 \\
.39 \\
.39 \\
.38\end{array}$ & $\begin{array}{l}430 \\
350 \\
260 \\
180 \\
100\end{array}$ \\
\hline $\begin{array}{r}850 \\
900 \\
950 \\
1000\end{array}$ & $\begin{array}{c}1.3 \\
1.1 \\
8.8 \times 10^{3} \\
7.4\end{array}$ & $\begin{array}{l}.37 \\
.36 \\
.36 \\
.35\end{array}$ & $\begin{array}{l}95 \\
80 \\
75 \\
70\end{array}$ \\
\hline
\end{tabular}

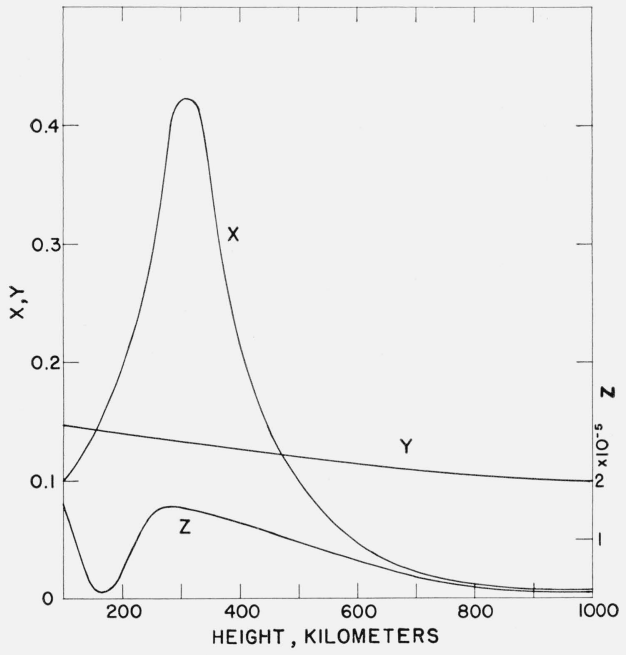

Figure 4. Normalized ionosphere parameter versus altitude (based on data in table 1); frequency $=10$ $M c / s$.

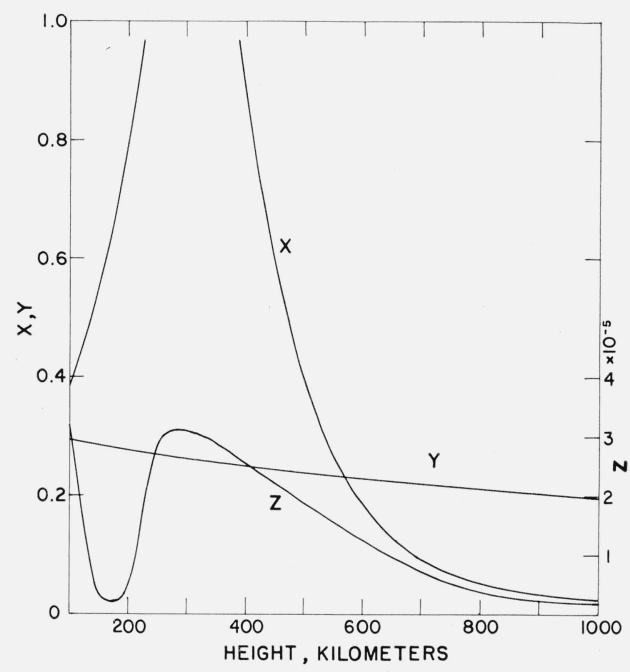

Figure 5. Normalized ionosphere parameter versus altitude (based on data in table 1); frequency $=5$ $\mathrm{Mc} / \mathrm{s}$.

unity or larger, so that the product $P$ in (101) is small or negative. For smaller and greater altitudes than this region, computations of $Z$ were made on the NASA 7090 computer. The resulting impedance, plotted against $\theta$, the angle of the dipole's axis with respect to the earth's magnetic field, is plotted for a number of altitudes in figures 6 and 7 for $10 \mathrm{Mc} / \mathrm{s}$, in figures 8,9 , and 10 for $5 \mathrm{Mc} / \mathrm{s}$, and for an altitude of $1000 \mathrm{~km}$ in figure 11 for $2 \mathrm{Mc} / \mathrm{s}$.

The antenna in question is a half-wave dipole of radius-to-half-length ratio $\epsilon=1 / 300$. Thus $\Lambda=k_{a} l$ has the value 1.5708 (i.e., $\pi / 2$ ). This corresponds to a situation where the length of the dipole is varied with altitude so that it would always have near-zero reactance. That is, the impedances calculated in figures 6 to 11 are based on the assumption of a sinusoidal current distribution of the form $\sin k_{a}(l-|z|)=\cos \left(k_{a} z\right), k_{a}$ being a real constant having the value $\sqrt{1-x} k_{0}$, independent of $\theta$, where the ionospheric parameter $x$ is that corresponding to the particular altitude and frequency. 


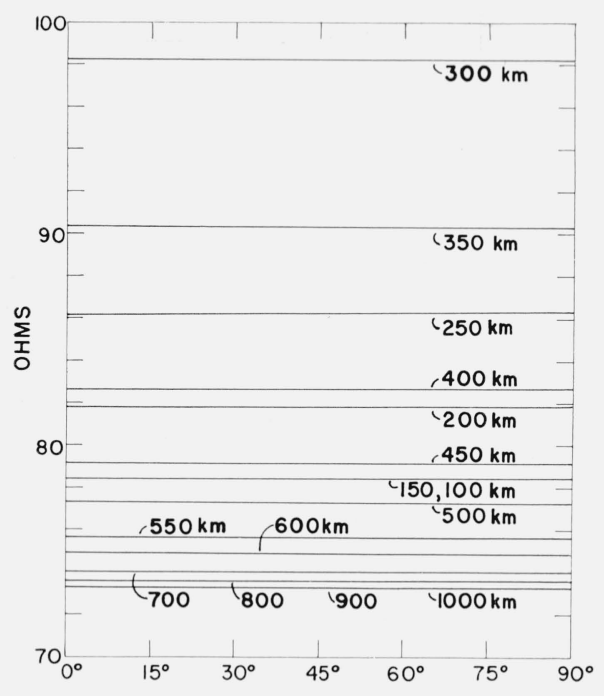

Figure 6. Resistance of half-wave dipole versus angle to magnetic field; frequency $=10 \mathrm{Mc} / \mathrm{s}$. Ionosphere parameters as in table 1.

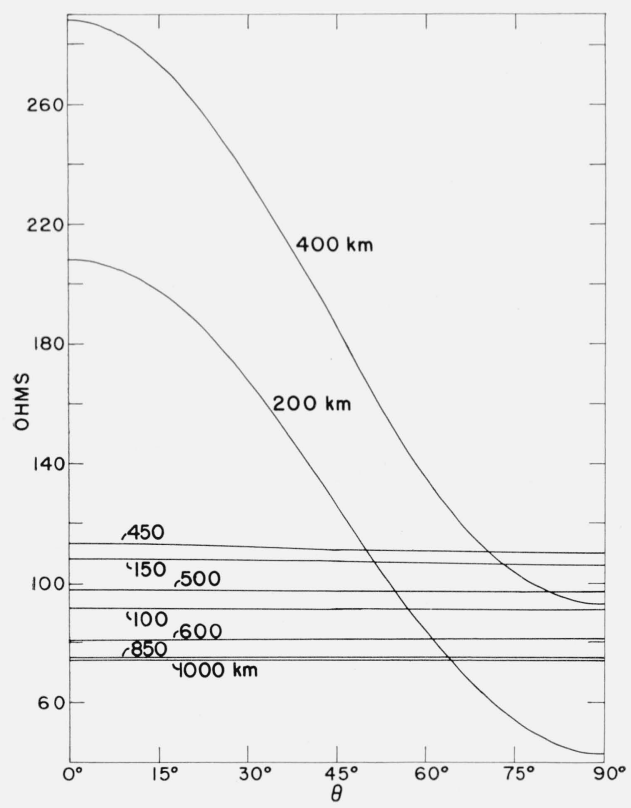

Figure 8. Resistance of half-wave dipole versus angle to magnetic field; frequency $=5 \mathrm{Mc} / \mathrm{s}$. Ionosphere parameters as in table 1.

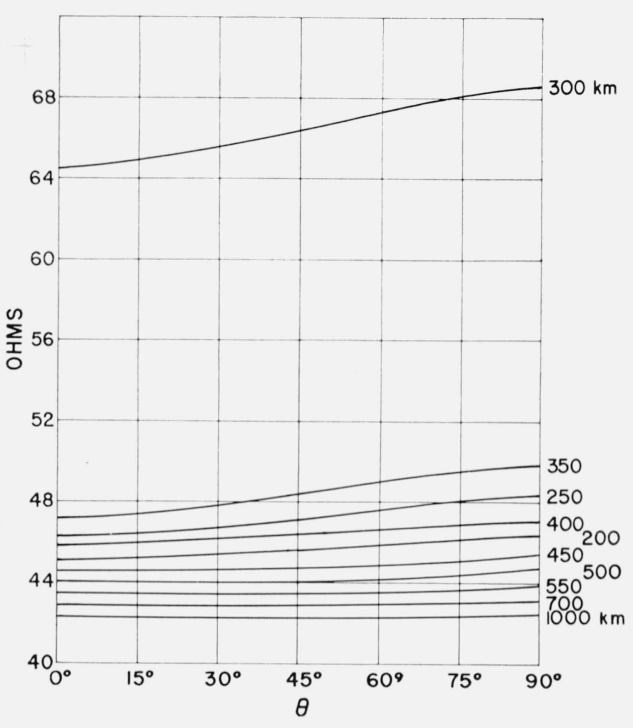

Figure 7. Reactance of half-wave dipole versus angle to magnetic field; frequency $=10 \mathrm{Mc} / \mathrm{s}$. Ionosphere parameters as in table 1 .

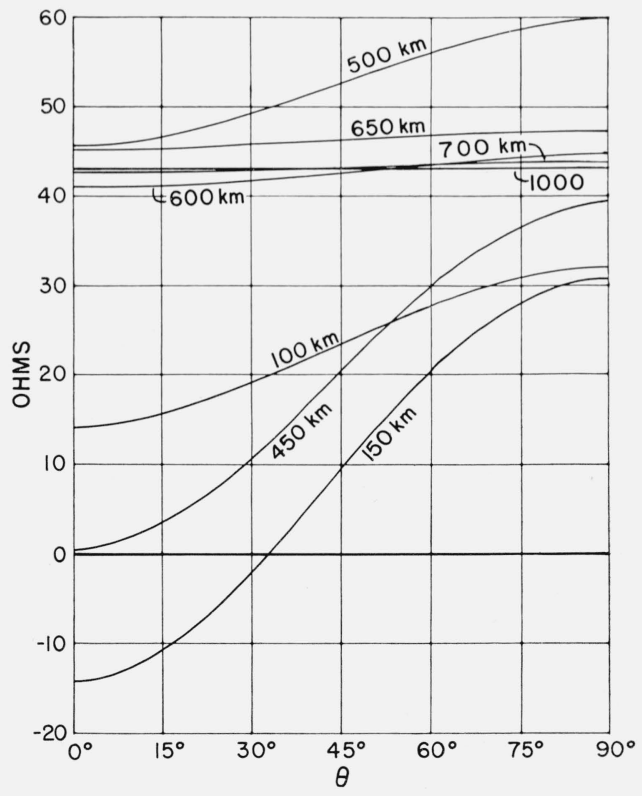

Figure 9. Reactance of half-wave dipole versus angle to magnetic field; frequency $=5 \mathrm{Mc} / \mathrm{s}$.

Ionosphere parameters as in table 1. 


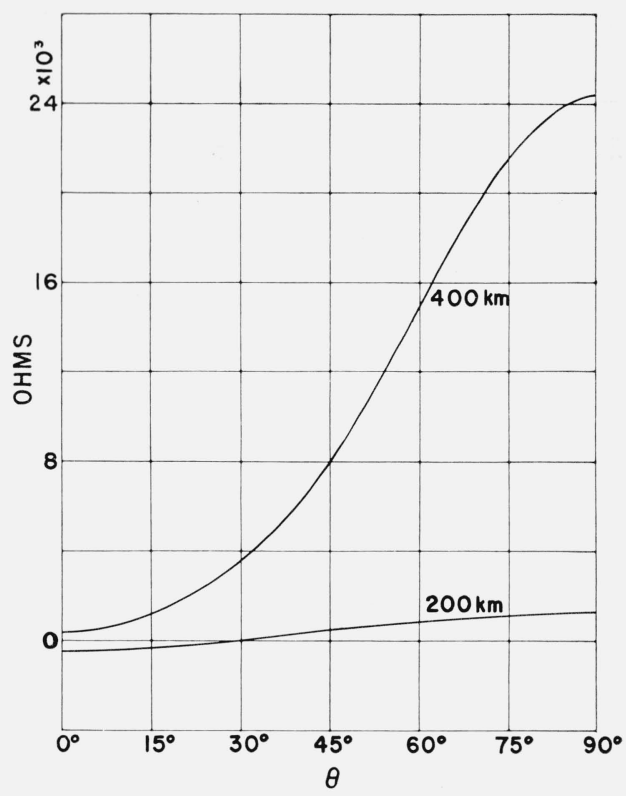

Figure 10. Reactance of half-wave dipole versus angle to magnetic field; frequency $=5 \mathrm{Mc} / \mathrm{s}$.

Ionosphere parameters as in table 1.

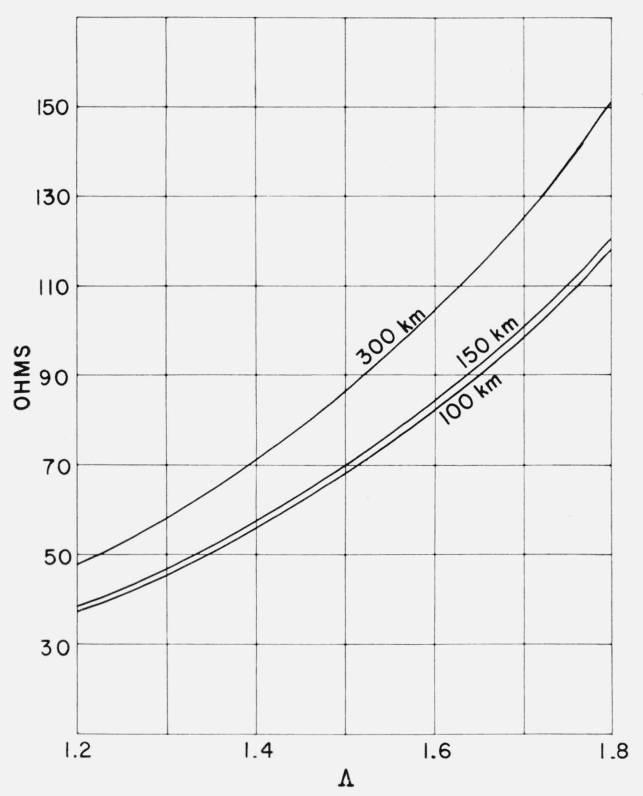

Figure 12. Dipole resistance versus $\Lambda=\mathrm{k}_{\mathrm{a}} \mathrm{l}$; frequency $=10 \mathrm{Mc} / \mathrm{s}, \theta=45^{\circ}$.

Ionosphere parameters as in table 1 .

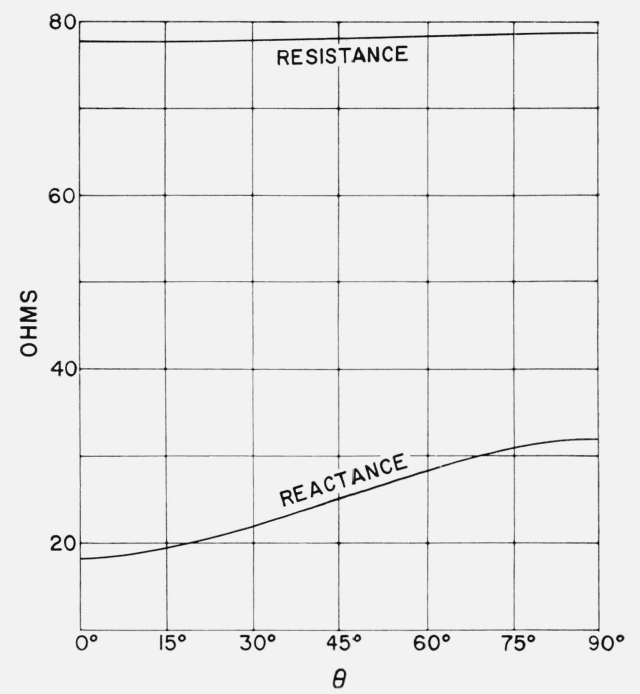

Figure 11. Impedance of half-wave dipole versus angle to magnetic field; frequency $=2 \mathrm{Mc} / \mathrm{s}$.

Ionosphere parameters as in table 1.

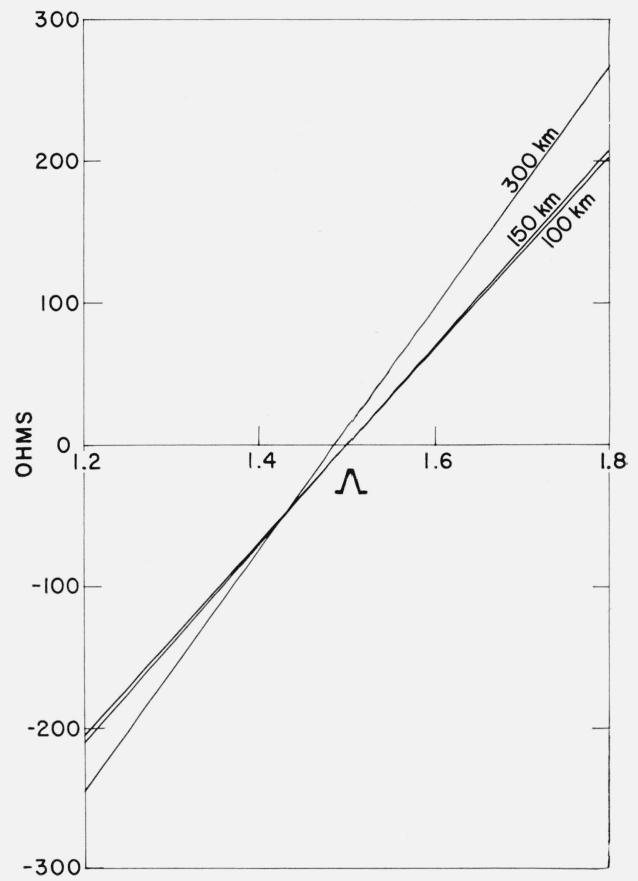

Figure 13. Dipole reactance versus $\Lambda=\mathrm{k}_{\mathrm{a}} \mathrm{l}$; frequency $=10 \mathrm{Mc} / \mathrm{s}, \theta=45^{\circ}$.

Ionosphere parameters as in table 1 . 


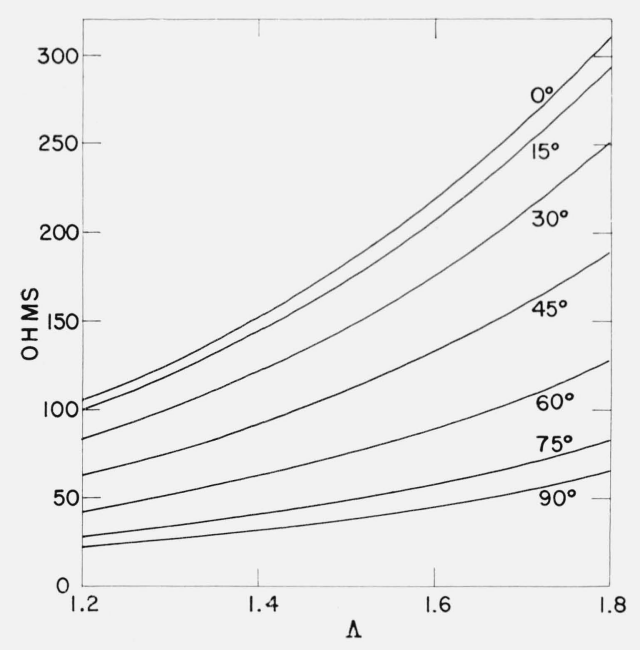

Figure 14. Dipole resistance versus $\Lambda=\mathrm{k}_{\mathrm{a}} \mathrm{l}$; frequency $=5 \mathrm{Mc} / \mathrm{s}$, altitude $=200 \mathrm{~km}$.

Ionosphere pacameters as in table 1.

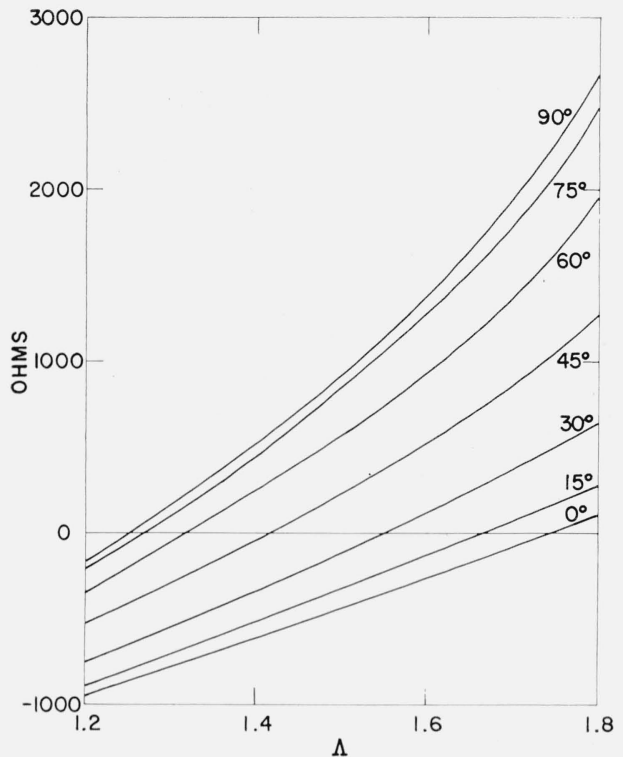

Figure 15. Dipole reactance versus $\Lambda=\mathrm{k}_{\mathrm{a}} \mathrm{l}$; frequency $=5 \mathrm{Mc} / \mathrm{s}$, altitude $=200 \mathrm{~km}$.

Ionosphere parameters as in table 1 .

In order to explore the magnitude of the change of impedance with physical length of the dipole, curves were also computed for the reactance and resistance as function of dipole length, for values of $\Lambda=k_{a} l$ near $\pi / 2$. The results are plotted here in figures 12 to 15 , for the special conditions listed in the legends.

In summarizing these plots, we first note that for $10 \mathrm{Mc} / \mathrm{s}$ and $1000 \mathrm{~km}$ altitude, where the ionospheric conditions have small effect and free-space conditions are being approached, the calculated impedance shown in figures 6 and 7 shows a negligible $\theta$-dependence about a value identical with that of a half-wave dipole in free space [Carter, 1932].

Next, it is obvious from the graphs that the impedance is markedly affected in the altitude range where the ionospheric parameter $x$ approaches unity. The impedance then becomes markedly dependent on angle, more especially the reactive component. This is especially noticeable in figure 10 for an altitude of $400 \mathrm{~km}$. When this curve was first obtained, it was felt that these large values may have been the result of the approach of the parameter $P$ of (101) to a small value (about 0.01 ) so that the numerical integration may have become unreliable. To check this, the 16-point numerical integration was changed to 32-point, but the computed results changed negligibly. This is highly encouraging as to the accuracy of the numerical integration process.

It should be pointed out that these curves are based on the assumptions stated previously, and therefore should not be taken as the best obtainable under the present theory. In particular, the $k_{a}$-value used here is real and independent of orientation angle, whereas the variational estimates of $k_{a}$ referred to at the end of section 6 would lead to a complex $\theta$-dependent value. But this refinement will be handled in a later treatment.

It should also be pointed out that the present theory assumes a homogeneous medium around the dipole. It therefore does not incorporate the effect of the plasma sheath which may be formed around the dipole in an actual case.

\section{Summary}

In this paper, we have formulated the problem of the impedance of a thin center-driven dipole in a magneto-ionic medium by expressing the Green's function as a Fourier integral in spherical propagation-constant coordinates. In performing a residues evaluation of the radial integral in this coordinate system, it is found necessary to deform the contour differently 
in different angular regions. By an appropriate rotation of the coordinates, the boundaries of these angular regions are made to depend on a single angular variable.

An expansion of the result in power series in the small parameter $\epsilon=$ (dipole radius/dipole half-length), in which only terms in $\log (1 / \epsilon)$ and $\epsilon^{0}$ are retained, leads to considerable simplification. It is found that the two dominant terms can be expressed as sine and cosine integrals, as in the free-space case, plus two finite single integrals which need numerical evaluation. The integrands of these latter integrals are expressed in terms of recursive routines.

The numerical integrations are in terms of the polar angle of the wave propagation vector with respect to the earth's magnetic field. Singularities of the integrand are encountered when the applied frequency is below the plasma or gyrofrequencies. It is then necessary to carry out the numerical integration in the complex plane. This has not been investigated in this paper. Consequently the present numerical integration procedure is restricted to cases where this situation does not occur. The closed-form terms, however, are valid in all parameter ranges.

Numerical calculations of impedance for selected values of the parameters are exhibited in a set of curves.

It is pointed out that the problem may be recast variationally so that the current distribution is computed rather than assumed. This approach, which requires no substantial changes in the present analytical and numerical procedures, will be given in a later paper.

\section{References}

Aitken, A. C. (1939), Statistical mathematics, ch. VI (Oliver and Boyd, Edinburgh and London, New York, Interscience Publishers, Inc.).

Bierens de Hahn, David (1957), Nouvelles tables d’integrales definites, (Hafner Publishing Co., New York, N.Y.).

Carter, P. S. (1932), Circuit relations in radiating systems and applications to antenna problems, Proc. IRE 20, 1016.

Jackson, J. E., and J. A. Kane (1959), Measurements of ionospheric electron densities using an RF probe technique, J. Geophys. Res. 64, 1074-1075.

Jahnke, E., and F. Emde (1945), Tables of functions, p. 3 (Dover Publications, New York, N.Y.).

King, R. W. P. (1956), The theory of linear antennas, (Harvard University Press, Cambridge, Mass.).

Kogelnik, H. (Sept.-Oct. 1960), On electromagnetic radiation in magneto-ionic media, J. Res. NBS 64D (Radio Prop.), No. 5, 515-523.

Seddon, J. C. (1953), Propagation measurements in the ionosphere with the aid of rockets, J. Geophys. Res. 58, $323-335$.

Storer, J. E. (Feb. 10, 1950), Variational solution to the problem of the symmetrical cylindrical antenna, Cruft Lab. Tech. Report No. 101, Harvard University, Cambridge, Mass.

Evaluate

\section{Appendix A}

$$
A_{2 n} \equiv \sum_{i=1}^{4} \frac{S_{i}^{2 n}}{\left(S_{i}^{2}-S_{j}^{2}\right)\left(S_{i}^{2}-S_{k}^{2}\right)\left(S_{i}^{2}-S_{l}^{2}\right)} \cdot
$$

The $S_{i}^{2}$ are roots of

$$
S_{i}^{8}-d_{1} S_{i}^{6}+d_{2} S_{i}^{4}-d_{3} S_{i}^{2}+d_{4}=0,
$$

where

$$
\begin{aligned}
& d_{1}=S_{1}^{2}+S_{2}^{2}+S_{3}^{2}+S_{4}^{2} \\
& d_{2}=S_{1}^{2} S_{2}^{2}+S_{1}^{2} S_{3}^{2}+S_{1}^{2} S_{4}^{2}+S_{2}^{2} S_{3}^{2}+S_{2}^{2} S_{4}^{2}+S_{3}^{2} S_{4}^{2} \\
& d_{3}=S_{1}^{2} S_{2}^{2} S_{3}^{2}+S_{1}^{2} S_{2}^{2} S_{4}^{2}+S_{2}^{2} S_{3}^{2} S_{4}^{2} \\
& d_{4}=S_{1}^{2} S_{2}^{2} S_{3}^{2} S_{4}^{2} .
\end{aligned}
$$


Consider the determinant

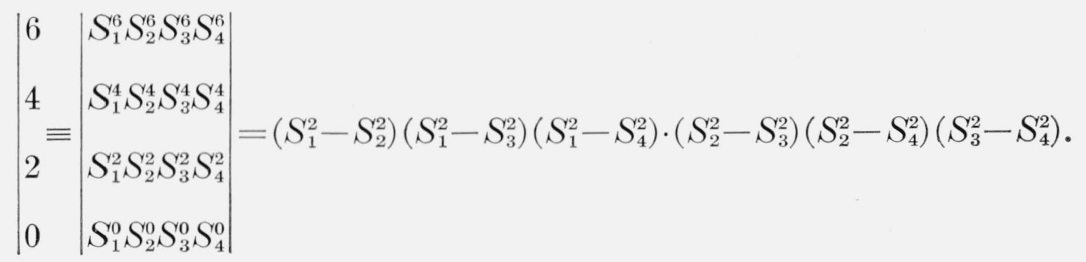

This is an alternating function [Aitken, 1939] of the $S_{i}, i=1, \ldots, 4$.

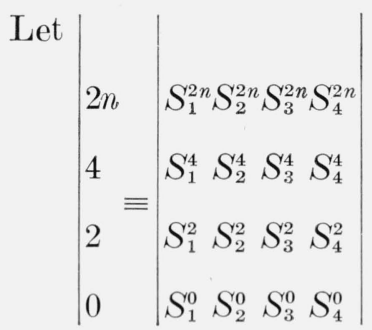

The four terms of $A_{2 n}$ in (A1) may be gathered over a common denominator which may be written in the form (A3). When this is done, it is seen that the resulting numerator is simply an expansion of the determinant in (A4). Thus

$$
A_{2 n}=\left|\begin{array}{l}
2 n \\
4 \\
2 \\
0
\end{array} \div \quad \div\right| \begin{aligned}
& 6 \\
& 4 \\
& 2 \\
& 0
\end{aligned}
$$

For $2 n=0,2$, or 4 , two rows of the determinant in the numerator are identical and the determinant vanishes, so that

$$
A_{0}=A_{2}=A_{4}=0 \text {. }
$$

For $2 n=6$, numerator and denominator are identical:

$$
A_{6}=1 .
$$

Rearranging (A2) and multiplying through by $S_{i}^{2 n-8}, n \geq 4$, we have

$$
S_{i}^{2 n}=S_{i}^{2 n}=d_{1} S_{i}^{2 n-2}-d_{2} S_{i}^{2 n-4}+d_{3} S_{i_{i}}^{2 n-6}-d_{4} S_{i}^{2 n-8}, \quad n \geq 4 .
$$

By (A1) or (A5) this implies

$$
A_{2 n}=A_{2 n}=d_{1} A_{2 n-2}-d_{2} A_{2 n-4}+d_{3} A_{2 n-6}-d_{4} A_{2 n-8}, \quad n \geq 4 .
$$

Equations (A6), (A7), (A8) provide the basis for recursive evaluation of the $A_{2 n}$ encountered in the text.

Evaluation of

\section{Appendix B}
(a) $f_{n}=\frac{1}{2 \pi} \int_{0}^{2 \pi}|a+b \sin \beta|^{n} d \beta$
(b) $g_{n}=\frac{1}{2 \pi} \int_{0}^{2 \pi} \cos ^{2} \beta|a+b \sin \beta|^{n} d \beta$. 
Define

$I_{n}(x, y|| a, b) \equiv \int_{x}^{y}(a+b \sin \beta)^{n} d \beta=a \int_{x}^{y}(a+b \sin \beta)^{n-1} d \beta+b \int_{x}^{y} \sin \beta(a+b \sin \beta)^{n-1} d \beta$

$=a I_{n-1}-\left.b \cos \beta(a+b \sin \beta)^{n-1}\right|_{x} ^{y}+b^{2}(n-1) \int_{x}^{y} \cos ^{2} \beta(a+b \sin \beta)^{n-2} d \beta$

$$
\cos ^{2} \beta=1-\sin ^{2} \beta=1-\frac{[(a+b \sin \beta)-a]^{2}}{b^{2}} .
$$

Hence

$$
\begin{aligned}
I_{n} & =a I_{n-1}-\left.b \cos \beta(a+b \sin \beta)^{n-1}\right|_{x} ^{y}+(n-1)\left(b^{2} I_{n-2}^{-}-I_{n}+2 a I_{n-1}-a^{2} I_{n-2}\right) \\
& =\frac{1}{n}\left\{(2 n-1) a I_{n-1}+(n-1)\left(b^{2}-a^{2}\right) I_{n-2}-\left.b \cos \beta(a+b \sin \beta)^{n-1}\right|_{x} ^{y}\right\} .
\end{aligned}
$$

(a)

$$
f_{n}=\frac{1}{2 \pi} \int_{0}^{2 \pi}|a+b \sin \beta|^{n} d \beta=\frac{1}{2 \pi} \int_{0}^{2 \pi}|\bar{a}+\bar{b} \sin \beta|^{n} d \beta
$$

where $\bar{a}=|a|, \bar{b}=|b|$.

Case (i): If $\bar{a} \geq \bar{b}$, then $a+b \sin \beta$ does not change $\operatorname{sign}$ in $(0,2 \pi)$, so that

Hence from (B1),

$$
\left.\bar{b} \cos \beta(\bar{a}+\bar{b} \sin \beta)^{n-1}\right|_{0} ^{2 \pi}=0 .
$$

$$
f_{n}=\frac{1}{n}\left[(2 n-1) \bar{a} f_{n-1}-(n-1)\left(a^{2}-b^{2}\right) f_{n-2}\right] .
$$

Case (ii): If $\bar{b}>\bar{a}$, put

$$
c=\sin ^{-1} \frac{\bar{a}}{\bar{b}} .
$$

Then

$$
\begin{aligned}
2 \pi f_{n} & =\int_{0}^{2 \pi}|\bar{a}+\bar{b} \sin \beta|^{n} d \beta=\int_{0}^{\pi}(\bar{a}+\bar{b} \sin \beta)^{n} d \beta+\int_{\pi}^{2 \pi}|\bar{a}+\bar{b} \sin \beta|^{n} d \beta \\
& =\int_{0}^{\pi}(\bar{a}+\bar{b} \sin \beta)^{n} d \beta+\int_{0}^{\pi}|\bar{a}-\bar{b} \sin \beta|^{n} d \beta \\
& =\int_{0}^{\pi}(\bar{a}+\bar{b} \sin \beta)^{n} d \beta+\int_{0}^{c}(\bar{a}-\bar{b} \sin \beta)^{n} d \beta+\int_{c}^{\pi-c}(-\bar{a}+\bar{b} \sin \beta)^{n} d \beta+\int_{\pi-c}^{\pi}(\bar{a}-\bar{b} \sin \beta)^{n} d \beta \\
& =2 \int_{0}^{\pi / 2}(\bar{a}+\bar{b} \sin \beta)^{n} d \beta+2 \int_{0}^{c}(\bar{a}-\bar{b} \sin \beta)^{n} d \beta+2 \int_{c}^{\pi-c}(-\bar{a}+\bar{b} \sin \beta)^{n} d \beta \\
& =2 I_{n}\left(0, \frac{\pi}{2} \mid \bar{a}, \bar{b}\right)+2 I_{n}(0, c \| \bar{a},-\bar{b})+2 I_{n}(c, \pi / 2 \|-\bar{a}, \bar{b}) \\
& \equiv 2 \pi\left(U_{n}+V_{n}\right)
\end{aligned}
$$

where

$$
\begin{aligned}
& 2 \pi U_{n}=2 I_{n}\left(0, \frac{\pi}{2} \| \bar{a}, \bar{b}\right)+2 I_{n}(0, c \|-\bar{a}, \bar{b}) \\
& 2 \pi V_{n}=2 I_{n}\left(c, \frac{\pi}{2} \|-\bar{a}, \bar{b}\right) .
\end{aligned}
$$


Applying (B1) to the right-hand side of (B3), we have for the last term of (B1) $\left.-\left.\bar{b} \cos \beta(\bar{a}+\bar{b} \sin \beta)^{n-1}\right|_{0} ^{\pi}+\left.2 \bar{b} \cos \beta(\bar{a}-\bar{b} \sin \beta)^{n-1}\right|_{0} ^{c}-\left.2 \bar{b} \cos \beta(-\bar{a}+\bar{b} \sin \beta)^{n-1}\right|_{c} ^{\pi / 2} \sin \beta\right)\left.^{n-1}\right|_{c} ^{\pi / 2}$

$$
\begin{array}{ll}
=2 \overline{b a}^{n-1} & -2 \overline{b a^{n}-1} \\
=0 . &
\end{array}
$$

Hence from (B3), (B4), and (B1) we obtain the recursion formula for $f n$ in case (ii):

$$
\begin{aligned}
f_{n} & =\frac{1}{n}\left\{(2 n-1) \bar{a} U_{n-1}+(n-1)\left(b^{2}-a^{2}\right) U_{n-2}\right\}-\frac{1}{n}\left\{(2 n-1) \bar{a} V_{n-1}-(n-1)\left(b^{2}-a^{2}\right) V_{n-2}\right\} \\
& =\frac{1}{n}\left\{(2 n-1) \bar{a}\left(U_{n-1}-V_{n-1}\right)+(n-1)\left(b^{2}-a^{2}\right) f_{n-2}\right\} .
\end{aligned}
$$

Also

$$
\begin{aligned}
U_{n} & =\frac{1}{n}\left[(n-1)\left(b^{2}-a^{2}\right) U_{n-2}+(2 n-1) \bar{a} U_{n-1}\right], \\
V_{n} & =\frac{1}{n}\left[(n-1)\left(b^{2}-a^{2}\right) V_{n-2}-(2 n-1) \bar{a} V_{n-1}\right] \\
U_{0} & =\frac{1}{2}+\frac{c}{\pi} \\
U_{1} & =\bar{a} U_{0}+\frac{1}{\pi}\left(b^{2}-a^{2}\right)^{1 / 2} \\
V_{0} & =\frac{1}{2}-\frac{c}{\pi} \\
V_{1} & =-\bar{a} V_{0}+\frac{1}{\pi}\left(b^{2}-a^{2}\right)^{1 / 2} .
\end{aligned}
$$

(b)

$$
g_{n}=\frac{1}{2 \pi} \int_{0}^{2 \pi} \cos ^{2} \beta|a+b \sin \beta|^{n} d \beta=\frac{1}{2 \pi} \int_{0}^{2 \pi} \cos ^{2} \beta\left|\bar{a}+\bar{b}_{-} \sin \beta\right|^{n} d \beta .
$$

\section{Consider}

$$
\begin{aligned}
J_{n}(x, y \| a, b) & =\int_{\chi}^{y} \cos ^{2} \beta(a+b \sin \beta)^{n} d \beta=\int_{\chi}^{y}\left(1-\sin ^{2} \beta\right)(a+b \sin \beta)^{n} d \beta \\
& =\int_{\chi}^{y}\left\{\frac{b^{2}-[(a+b \sin \beta)-a]^{2}}{b^{2}}\right\}(a+b \sin \beta)^{n} d \beta \\
& =\frac{1}{b^{2}}\left[\left(b^{2}-a^{2}\right) I_{n}+2 a I_{n+1}-I_{n+2}\right] .
\end{aligned}
$$

Then we have

Case (i) $\bar{a} \geq \bar{b}$ :

$$
g_{n}=\frac{1}{b^{2}}\left[\left(b^{2}-a^{2}\right) f_{n}+2 \bar{a} f_{n+1}-f_{n+2}\right]
$$

Case (ii) $\bar{b}>\bar{a}$ :

$$
g_{n}=\frac{1}{b^{2}}\left[\left(b^{2}-a^{2}\right)\left(U_{n}+V_{n}\right)+2 \bar{a}\left(U_{n+1}-V_{n+1}\right)-\left(U_{n+2}+V_{n+2}\right)\right] .
$$

(Paper 68D4-355) 